\title{
DYSON'S NEW SYMMETRY AND GENERALIZED ROGERS-RAMANUJAN IDENTITIES
}

\author{
CILANNE BOULET*
}

\begin{abstract}
We present a generalization, which we call $(k, m)$-rank, of Dyson's notion of rank to integer partitions with $k$ successive Durfee rectangles and give two combinatorial symmetries associated with this new definition. We prove these symmetries bijectively. Using the two symmetries we give a new combinatorial proof of generalized Roger-Ramanujan identities. We also describe the relationship between $(k, m)$-rank and Garvan's $k$-rank.
\end{abstract}

\section{INTRODUCTION}

First discovered by Rogers [22] in 1894, the Rogers-Ramanujan identities,

$$
\sum_{n=0}^{\infty} \frac{q^{n^{2}}}{(1-q)\left(1-q^{2}\right) \cdots\left(1-q^{n}\right)}=\prod_{n=0}^{\infty} \frac{1}{\left(1-q^{5 n+1}\right)\left(1-q^{5 n+4}\right)}
$$

and

$$
\sum_{n=0}^{\infty} \frac{q^{n^{2}+n}}{(1-q)\left(1-q^{2}\right) \cdots\left(1-q^{n}\right)}=\prod_{n=0}^{\infty} \frac{1}{\left(1-q^{5 n+2}\right)\left(1-q^{5 n+3}\right)},
$$

are among the most intriguing partition identities.

The goal of this paper is to present a new combinatorial proof of the following generalization (which is due to Andrews [2]) of the first Rogers-Ramanujan identity, for $k \geq 1$ :

$$
\sum_{n_{1}=0}^{\infty} \cdots \sum_{n_{k-1}=0}^{\infty} \frac{q^{N_{1}^{2}+N_{2}^{2}+\cdots+N_{k-1}^{2}}}{(q)_{n_{1}}(q)_{n_{2}} \cdots(q)_{n_{k-1}}}=\prod_{\substack{n=1 \\ n \neq 0, \pm k(\bmod 2 k+1)}}^{\infty} \frac{1}{1-q^{n}}
$$

where $N_{j}=n_{j}+n_{j+1}+\cdots+n_{k-1}$. We use the standard $q$-series notation and let $(q)_{\infty}=\prod_{i=1}^{\infty}\left(1-q^{i}\right)$ and $(q)_{n}=\prod_{i=1}^{n}\left(1-q^{i}\right)$.

Instead of attacking this identity directly, we will use two bijections to prove the following family of identities, which we call the generalized Schur identities:

$$
\sum_{n_{1}=0}^{\infty} \cdots \sum_{n_{k-1}=0}^{\infty} \frac{q^{N_{1}^{2}+N_{2}^{2}+\cdots+N_{k-1}^{2}}}{(q)_{n_{1}}(q)_{n_{2}} \ldots(q)_{n_{k-1}}}=\frac{1}{(q)_{\infty}} \sum_{j=-\infty}^{\infty}(-1)^{j} q^{\frac{j(j+1)(2 k+1)}{2}-k j},
$$

with $N_{j}=n_{j}+n_{j+1}+\cdots+n_{k-1}$. By using Jacobi's triple product identity,

$$
\sum_{j=-\infty}^{\infty} z^{j} t^{\frac{j(j+1)}{2}}=\prod_{i=1}^{\infty}\left(1+z t^{i}\right) \prod_{j=0}^{\infty}\left(1+z^{-1} t^{j}\right) \prod_{i=1}^{\infty}\left(1-t^{i}\right)
$$

Key words and phrases. Rogers-Ramanujan identity, Schur's identity, successive Durfee squares, Dyson's rank, bijection, integer partition.

*Department of Mathematics, Cornell University, Ithaca, NY 14853. Email: cilanne@math.cornell.edu. 
which specializes to

$$
\sum_{j=-\infty}^{\infty}(-1)^{j} q^{\frac{j(j+1)(2 k+1)}{2}-k j}=\prod_{\substack{n=1 \\ n \equiv 0, \pm k(\bmod 2 k+1)}}^{\infty} 1-q^{n}
$$

when we let $t=q^{2 k+1}$ and $z=-q^{-k}$, we see that (11) and (2) are equivalent. This application of Jacobi's triple product identity is a standard first step in Rogers-Ramanujan proofs and in particular was used by Schur [23] in his combinatorial proof of the original Rogers-Ramanujan identities. We note that the Jacobi triple product identity has a combinatorial proof due to Sylvester (see [21, 25]) and so its application does not change the combinatorial nature of our proof.

Before presenting our proof of the generalized Rogers-Ramanujan identities (11), we must outline our notation and review two important ideas. The first is Andrews' notion of successive Durfee squares which gives a combinatorial interpretation to the left hand side of (11) and (2). The second is Dyson's proof of Euler's pentagonal number theorem based on his definition of rank.

1.1. Notation. We begin by giving the basic definitions that we will need. This section is meant simply to familiarize the reader with the notation that will be used, rather than provide an introduction to the subject. For such an introduction we recommend [3, 21].

A partition $\lambda$ is a sequence of integers $\left(\lambda_{1}, \lambda_{2}, \ldots, \lambda_{\ell}\right)$ such that $\lambda_{1} \geq \lambda_{2} \geq \ldots \geq \lambda_{\ell}>0$. As a convention, we will say that $\lambda_{j}=0$ for $j>\ell$. We call each $\lambda_{i}$ a part of $\lambda$. We say that $\lambda$ is a partition of $n$, denoted $\lambda \vdash n$ or $|\lambda|=n$, if $\sum \lambda_{i}=n$. Let $\mathcal{P}_{n}$ denote the set of partitions of $n$ and let $p(n)=\left|\mathcal{P}_{n}\right|$. Also, let $\mathcal{P}=\cup_{n} \mathcal{P}_{n}$ denote the set of all partitions.

We let $\ell(\lambda)=\ell$ denote the number of non-zero parts of $\lambda$. In general, we will let number of part mean number of non-zero parts. Let $f(\lambda)=\lambda_{1}$ denote the largest part of $\lambda$, and let $e(\lambda)=\lambda_{\ell(\lambda)}$ denote the smallest (non-zero) part of $\lambda$.

To every partition we associate a Young diagram as in Figure 1
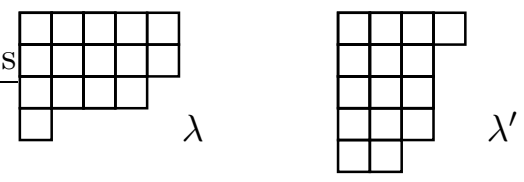

Figure 1. Partition $\lambda=(5,5,4,1)$ and conjugate partition $\lambda^{\prime}=(4,3,3,3,2)$.

The conjugate $\lambda^{\prime}$ of a partition $\lambda$ is obtained by reflection across the main diagonal (again see Figure (1). Alternatively, $\lambda^{\prime}$ may be defined as follows: $\lambda^{\prime}=\left(\lambda_{1}^{\prime}, \lambda_{2}^{\prime}, \ldots, \lambda_{f(\lambda)}^{\prime}\right)$ where $\lambda_{i}^{\prime}=\mid\left\{j: \lambda_{j} \geq\right.$ $i\} \mid$ is the number of parts of $\lambda$ which are greater than or equal to $i$.

1.2. Andrews' successive Durfee squares. Andrews introduced the idea of successive Durfee squares to study his generalized Rogers-Ramanujan identities 4]. He interpreted the left hand sides of equations (11) and (2) as follows.

Definition 1.1. The first Durfee square of a partition $\lambda$ is the largest square that fits in the upper left hand corner of the diagram of $\lambda$. The second Durfee square is the largest square that fit in the diagram of $\lambda$ below the first Durfee square of $\lambda$. In general, the $k$ th Durfee square is the largest square that fits below the $(k-1)$ st Durfee square of $\lambda$. 

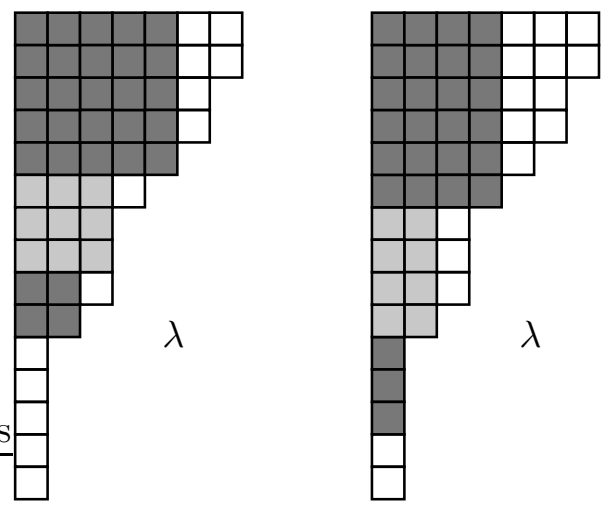

Figure 2. The first three successive Durfee squares and 1-Durfee rectangles of $\lambda=(7,7,6,6,5,4,3,3,3,2,1,1,1,1,1)$. On the left we see that $\lambda$ has successive Durfee squares of size 5,3 , and 2 . On the right we see that $\lambda$ has successive 1Durfee rectangles of width 4,2 , and 1 .

See Figure 2 for an example.

Let $q_{k}(n)$ denote the number of partitions of $n$ with at most $k$ Durfee squares and let $\mathcal{Q}_{k}$ denote the set of all partitions with at most $k$ Durfee squares. Now the generating function for partitions with Durfee squares of size $N_{1}, N_{2}, \ldots, N_{k-1}$ and no part below the $k-1$ st Durfee square is

$$
\frac{q^{N_{1}^{2}+N_{2}^{2}+\cdots+N_{k-1}^{2}}}{(q)_{n_{1}}(q)_{n_{2}} \cdots(q)_{n_{k-1}}}
$$

where $n_{j}=N_{j}-N_{j+1}$ so that $N_{j}=n_{j}+n_{j+1}+\cdots+n_{k-1}$. This can be seem by a simple counting argument as is done by Andrews [4]. Alternatively, in Appendix A of [7, we show this bijectively using the insertion procedure which is defined in this paper.

Therefore the generating function for partitions with at most $k-1$ Durfee squares is

$$
1+\sum_{n=1}^{\infty} q_{k-1}(n) q^{n}=\sum_{n_{1}=0}^{\infty} \cdots \sum_{n_{k-1}=0}^{\infty} \frac{q^{N_{1}^{2}+N_{2}^{2}+\cdots+N_{k-1}^{2}}}{(q)_{n_{1}}(q)_{n_{2}} \ldots(q)_{n_{k-1}}}
$$

with $N_{j}=n_{j}+n_{j+1}+\cdots+n_{k-1}$ which is indeed the left hand side of (11).

For our proof, we extend the notion of successive Durfee squares.

Definition 1.2. For any integer $m$, define an $m$-rectangle to be a rectangle whose height exceeds its width by exactly $m$. We require $m$-rectangles to have non-zero height though they may have width zero.

In particular, notice that 0-rectangles are simply squares. The technical detail about zero width being allowed will be used to obtain Observation 4.2

We define successive $m$-Durfee rectangles in the same manner as Andrews' successive Durfee squares.

Definition 1.3. The first $m$-Durfee rectangle of a partition $\lambda$ is the largest $m$-rectangle that fits in the upper left hand corner of the diagram of $\lambda$. The second $m$-Durfee rectangle is the largest $m$-rectangle that fits in the diagram of $\lambda$ below the first Durfee square of $\lambda$. In general, the $k$ th successive $m$-Durfee rectangle is the largest $m$-rectangle that fits below the $(k-1)$ st Durfee square of $\lambda$. 
Again, see Figure 2 for an example of successive 1-Durfee rectangles.

Note that the possibility of width zero $m$-rectangles means that, for $m>0$, all partitions (including the empty partition) have arbitrarily many successive $m$-Durfee rectangles. In this case, the Durfee rectangles extend below the non-zero parts of the partition.

1.3. Dyson's proof of Euler's pentagonal number theorem. The primary inspiration for the Rogers-Ramanujan proof in this paper is Dyson's proof of Euler's pentagonal number theorem,

$$
1=\frac{1}{(q)_{\infty}} \sum_{j=-\infty}^{\infty}(-1)^{j} q^{\frac{j(3 j-1)}{2}},
$$

based on his definition of rank [16] (see also [17]). Note that this identity is the case $k=1$ of the generalised Schur identities (2).

Definition 1.4 (Dyson [15]). The rank of a partition $\lambda$ is

$$
r(\lambda)=f(\lambda)-\ell(\lambda)
$$

Recall that $f(\lambda)$ is the length of the first part of $\lambda$ and $\ell(\lambda)$ is the number of parts of $\lambda$.

Dyson's proof of Euler's pentagonal number theorem can be presented as follows. (In addition to his papers, see [6] and [20] for additional descriptions.) Let $h(n, r), h(n, \leq r)$, and $h(n, \geq r)$ denote the number of partitions of $n$ with rank equal to $r$, less than or equal to $r$, and greater than or equal to $r$ respectively. Clearly, for $n>0$, we observe that

$$
p(n)=h(n, \leq r)+h(n, \geq r+1)
$$

and Dyson noticed two symmetries,

$$
\begin{aligned}
h(n, r) & =h(n,-r) \text { and } \\
h(n, \leq r) & =h(n+r-1, \geq r-2) .
\end{aligned}
$$

The first of these symmetries is a simple consequence of conjugation. The second symmetry, the "new symmetry" from the title of [16], follows from a bijection, $d_{r}$, which we call Dyson's map. Dyson's map $d_{r}$ takes a partition $\lambda$ of $n$ with $r(\lambda) \leq r$ and returns a partition $\mu$ of $n+r-1$ with $r(\mu) \geq r-2$ by removing the first column of $\lambda$, which has $\ell(\lambda)$ squares, and adding a part of size $\ell(\lambda)+r-1$. This new part will be the first row of $\mu$. See figure 3 for an example.
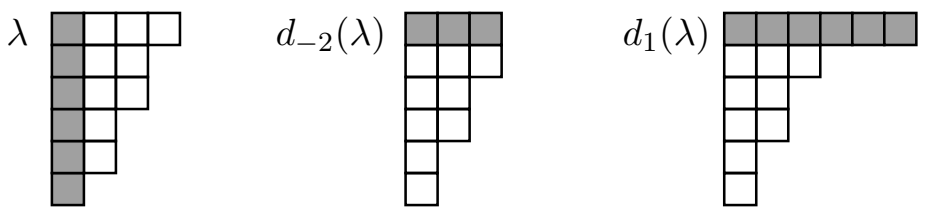

Figure 3. Applying Dyson's map to $\lambda=(4,3,3,2,2,1)$ with $r(\lambda)=4-6=-2$ gives $d_{-2}(\lambda)=(3,3,2,2,1,1)$ and $d_{1}(\lambda)=(6,3,2,2,1,1)$.

Let

$$
\begin{aligned}
& H_{\leq r}(q)=\sum_{n=1}^{\infty} h(n, \leq r) q^{n} \text { and } \\
& H_{\geq r}(q)=\sum_{n=1}^{\infty} h(n, \geq r) q^{n}
\end{aligned}
$$


be the generating functions for partitions with rank at most $r$ and at least $r$. Then

$$
H_{\leq r}(q)=q^{1-r} H_{\geq r-2}(q)=q^{1-r}\left(\frac{1}{(q)_{\infty}}-H_{\leq r-3}(q)\right)
$$

where the first equality follows from Dyson's new symmetry and the second equality follows from the observation. Applying this equation repeatedly gives

$$
\begin{aligned}
H_{\leq r}(q) & =q^{1-r}\left(\frac{1}{(q)_{\infty}}-H_{\leq r-3}(q)\right) \\
& =q^{1-r}\left(\frac{1}{(q)_{\infty}}\right)-q^{5-2 r}\left(\frac{1}{(q)_{\infty}}-H_{\leq r-6}(q)\right) \\
= & q^{1-r}\left(\frac{1}{(q)_{\infty}}\right)-q^{5-2 r}\left(\frac{1}{(q)_{\infty}}\right)+q^{12-3 r}\left(\frac{1}{(q)_{\infty}}-H_{\leq r-9}(q)\right) \\
& \vdots \\
= & \frac{1}{(q)_{\infty}} \sum_{j=1}^{\infty}(-1)^{j-1} q^{\frac{j(3 j-1)}{2}}-j r .
\end{aligned}
$$

Finally, the first symmetry (conjugation) gives us

$$
\frac{1}{(q)_{\infty}}=1+H_{\leq 0}(q)+H_{\geq 1}(q)=1+H_{\leq 0}(q)+H_{\leq-1}(q)
$$

and substituting gives Euler's pentagonal number theorem.

1.4. Outline of our proof. Roughly speaking, our proof of the generalized Schur identities is a Dyson-style proof with a modified Dyson's rank. In section 2] we develop the basic tools needed for our proof, selection and insertion. In section 3. we generalized Dyson's rank to partitions with $k$ successive $m$-Durfee rectangles. This new rank will be called $(k, m)$-rank. The definition will use the selection procedure from section 2. Similarly to the case of Dyson's rank, $(k, m)$-rank will satisfy two symmetries. We prove these in section 4 by two bijections that are build using using selection and insertion. The first bijection generalizes conjugation and the second bijection generalizes the map $d_{r}$ which corresponds to Dyson's new symmetry. In section 5. we use the same algebraic manipulations used to deduce Euler's pentagonal number theorem to deduce the generalized Schur identities. We conclude by explaining how our work relates to the work of others and by mentioning a problem that is still open.

\section{Basic Tools: Selection And Insertions}

In this section, we develop the basic tools that will be needed for our proof of the generalized Schur identities (21). We define two procedures which we call selection and insertion and we will say precisely in what sense they are inverses of each other.

2.1. Selection of parts from a sequence of partitions $\lambda^{1}, \lambda^{2}, \ldots, \lambda^{k}$. The first procedure, selection, has as input a sequence of partitions and as output one part from each of these partitions.

Procedure 2.1. Given a sequence of $k-1$ nonnegative integers,

$$
p_{2}, p_{3}, \ldots, p_{k},
$$


and $k$ partitions,

$$
\lambda^{1}, \lambda^{2}, \ldots, \lambda^{k}
$$

such that

$$
f\left(\lambda^{2}\right) \leq p_{2}, f\left(\lambda^{3}\right) \leq p_{3}, \ldots, f\left(\lambda^{k}\right) \leq p_{k},
$$

we select one row from each partition as follows:

- select the first (that is, the largest) part of $\lambda^{k}$,

- suppose we have selected the $j$ th part, $\lambda^{i}{ }_{j}$, of $\lambda^{i}$, then select the $\left(j+p_{i}-\lambda^{i}{ }_{j}\right)$ th part of $\lambda^{i-1}$.

One way to think of the selection of the $\left(j+p_{i}-\lambda_{j}^{i}\right)$ th part of $\lambda^{i-1}$ is to this that we are selecting the row of $\lambda^{i-1}$ that is $p_{i}-\lambda_{j}^{i}$ lower in the Young diagram than the row selected in $\lambda^{i}$. The number $p_{i}-\lambda_{j}^{i}$ can be thought of as the number of "missing" boxes in the $j$ th row of $\lambda^{i}$ since $\lambda^{i}$ is restricted to having parts of size at most $p_{i}$.

Definition 2.2. Let $A\left(\lambda^{1}, \lambda^{2}, \ldots, \lambda^{k} ; p_{2}, p_{3}, \ldots, p_{k}\right)$ be the sum of the selected parts.

When $p_{2}, p_{3}, \ldots, p_{k}$ are clear from the context, we will write $A\left(\lambda^{1}, \lambda^{2}, \ldots, \lambda^{k}\right)$.

See Figure 4 for examples of this selection procedure. On the left hand side, we have $p_{2}=4, p_{3}=2$, and $p_{4}=3$. We select the first part of $\lambda^{4}$. Then we select the $1+\left(p_{4}-\lambda_{1}^{4}\right)=1+(3-2)=2$ nd part from $\lambda^{3}$, the $2+\left(p_{3}+\lambda_{2}^{3}\right)=2+(2-2)=2$ nd part from $\lambda^{2}$, and the $2+\left(p_{2}+\lambda_{2}^{2}\right)=2+(4-2)=4$ th part from $\lambda^{1}$. This gives $A\left(\lambda^{1}, \lambda^{2}, \lambda^{3}, \lambda^{4}\right)=1+2+2+2=7$.

On the right hand side, we have $p_{2}=2, p_{3}=0, p_{4}=2$, and $p_{5}=6$. We select the first part of $\mu^{5}$. Then we select the $1+(6-6)=1$ st part from $\mu^{4}$, the $1+(2-0)=3$ th part from $\mu^{3}$, the $3+(0-0)=3$ th part from $\mu^{2}$, and the $3+(2-0)=5$ th part from $\mu^{1}$. This gives $A\left(\mu^{1}, \mu^{2}, \mu^{3}, \mu^{4}, \mu^{5}\right)=1+0+0+0+6=7$.
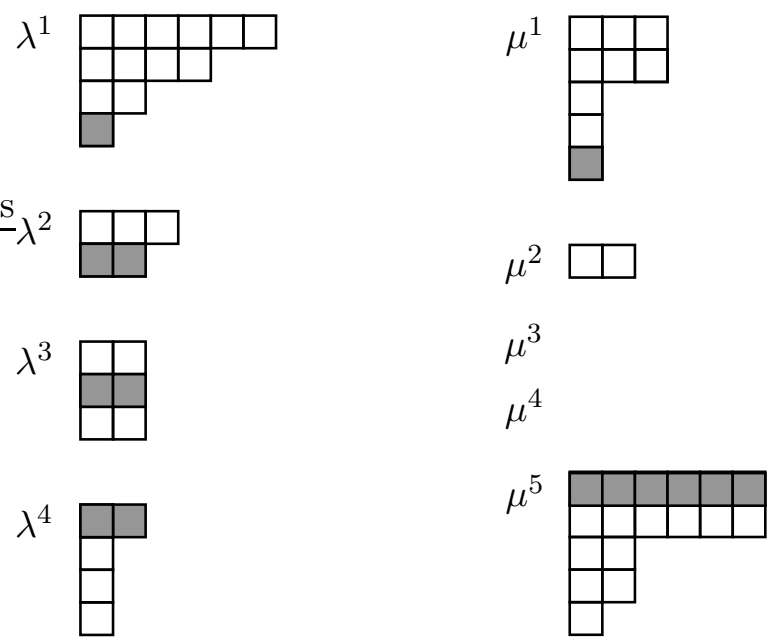

Figure 4. Selection of rows from $\lambda^{1}, \lambda^{2}, \lambda^{3}$, and $\lambda^{4}$ with $p_{2}=4, p_{3}=2$, and $p_{4}=3$ to get $A\left(\lambda^{1}, \lambda^{2}, \lambda^{3}, \lambda^{4}\right)=7$. Selection of rows from $\mu^{1}, \mu^{2}, \mu^{3}, \mu^{4}$, and $\mu^{5}$ with $p_{2}=2, p_{3}=0, p_{4}=2$, and $p_{5}=6$ to get $A\left(\mu^{1}, \mu^{2}, \mu^{3}, \mu^{4}, \mu^{5}\right)=7$. Selected parts are shown in grey.

It will be useful to establish notation for the selection of parts from a sequence of partitions and the removal of those parts. 
Definition 2.3. Given a sequence of $k-1$ nonnegative integers,

$$
p_{2}, p_{3}, \ldots, p_{k},
$$

and $k$ partitions,

$$
\lambda^{1}, \lambda^{2}, \ldots, \lambda^{k}
$$

such that

$$
f\left(\lambda^{2}\right) \leq p_{2}, f\left(\lambda^{3}\right) \leq p_{3}, \ldots, f\left(\lambda^{k}\right) \leq p_{k},
$$

- let $\psi_{1}=\psi_{1\left\{p_{2}, \ldots, p_{k}\right\}}\left(\lambda^{1}, \lambda^{2}, \ldots, \lambda^{k}\right)=A\left(\lambda^{1}, \lambda^{2}, \ldots, \lambda^{k}\right)$ and

- let $\psi_{2}=\psi_{2\left\{p_{2}, \ldots, p_{k}\right\}}\left(\lambda^{1}, \lambda^{2}, \ldots, \lambda^{k}\right)=\left(\mu^{1}, \mu^{2}, \ldots, \mu^{k}\right)$ where $\mu^{1}, \mu^{2}, \ldots, \mu^{k}$ are found by removing the parts of $\lambda^{1}, \lambda^{2}, \ldots, \lambda^{k}$ selected while calculating $\psi_{1}$.

Let $\psi_{\left\{p_{2}, \ldots, p_{k}\right\}}\left(\lambda^{1}, \lambda^{2}, \ldots, \lambda^{k}\right)=\left(\psi_{1} ; \psi_{2}\right)$.

When $\left\{p_{2}, \ldots, p_{k}\right\}$ are clear from context, we will write $\psi_{1}\left(\lambda^{1}, \lambda^{2}, \ldots, \lambda^{k}\right), \psi_{2}\left(\lambda^{1}, \lambda^{2}, \ldots, \lambda^{k}\right)$, and $\psi\left(\lambda^{1}, \lambda^{2}, \ldots, \lambda^{k}\right)$.

2.2. Insertion into a sequence of partitions $\lambda^{1}, \lambda^{2}, \ldots, \lambda^{k}$. Based on this definition of selection from a sequence of partitions, we can define an insertion algorithm on which our two symmetries are based. The following proposition describes the result of insertion. We will give a procedure for insertion after the proof of this proposition.

Proposition 2.4. Given a sequence of $k-1$ nonnegative integers

$$
p_{2}, p_{3}, \ldots, p_{k},
$$

$k$ partitions

$$
\lambda^{1}, \lambda^{2}, \ldots, \lambda^{k}
$$

with $\left|\lambda^{1}\right|+\left|\lambda^{2}\right|+\ldots+\left|\lambda^{k}\right|=n$, such that

$$
f\left(\lambda^{2}\right) \leq p_{2}, f\left(\lambda^{3}\right) \leq p_{3}, \ldots, f\left(\lambda^{k}\right) \leq p_{k},
$$

and an integer $a \geq A\left(\lambda^{1}, \lambda^{2}, \ldots, \lambda^{k} ; p_{2}, p_{3}, \ldots, p_{k}\right)$,

there exists a unique sequence of $k$ partitions,

$$
\mu^{1}, \mu^{2}, \ldots, \mu^{k},
$$

obtained by inserting one (possibly empty) part into each of the original partitions,

$$
\lambda^{1}, \lambda^{2}, \ldots, \lambda^{k},
$$

such that

(1) $\left|\mu^{1}\right|+\left|\mu^{2}\right|+\ldots+\left|\mu^{k}\right|=n+a$,

(2) $f\left(\mu^{2}\right) \leq p_{2}, f\left(\mu^{3}\right) \leq p_{3}, \ldots, f\left(\mu^{k}\right) \leq p_{k}$ ，

(3) $A\left(\mu^{1}, \mu^{2}, \ldots, \mu^{k} ; p_{2}, p_{3}, \ldots, p_{k}\right)=a$.

Moreover, the inserted parts have the same length as those which are selected when calculating $A\left(\mu^{1}, \mu^{2}, \ldots, \mu^{k} ; p_{2}, p_{3}, \ldots, p_{k}\right)$.

We will prove this proposition by induction on $a$. The two following lemmas are the required base case and inductive step.

Lemma 2.5. Proposition 2.4 (without uniqueness) is true for $a=A\left(\lambda^{1}, \lambda^{2}, \ldots, \lambda^{k} ; p_{2}, p_{3}, \ldots, p_{k}\right)$. 
Proof. For each $\lambda^{i}$, consider the size of the part selected from that partition. Insert an additional part in $\lambda^{i}$ of the same size as the selected part to obtain $\mu^{i}$. See Figure 5 .
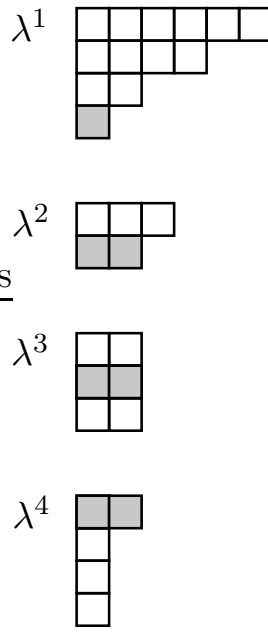
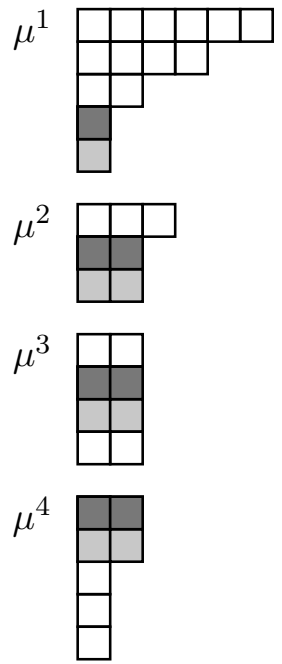

FIgURE 5. With $p_{2}=4, p_{3}=2$, and $p_{4}=3$, inserting $7=A\left(\lambda^{1}, \lambda^{2}, \lambda^{3}, \lambda^{4}\right)$ into $\lambda^{1}$, $\lambda^{2}, \lambda^{3}$, and $\lambda^{4}$ gives $\mu^{1}, \mu^{2}, \mu^{3}$, and $\mu^{4}$.

We have inserted parts totaling $a=A\left(\lambda^{1}, \lambda^{2}, \ldots, \lambda^{k} ; p_{2}, p_{3}, \ldots, p_{k}\right)$ since the sum of the selected parts of $\lambda^{1}, \lambda^{2}, \ldots, \lambda^{k}$ is $a$. This implies condition (1).

Note that, for each $\lambda^{i}$, since we are inserting a part of the same size as the selected part, it can be inserted directly above the selected row in $\lambda^{i}$. Again since we are inserting parts of the same size and since $p_{2}, p_{3}, \ldots, p_{k}$ remain constant, when we select rows from $\mu^{1}, \mu^{2}, \ldots, \mu^{k}$, we will select the rows we have just added. Moreover, this gives $A\left(\mu^{1}, \mu^{2}, \ldots, \mu^{k} ; p_{2}, p_{3}, \ldots, p_{k}\right)=a$, condition (3).

Finally, condition (2) is satisfied since $f\left(\lambda^{i}\right) \leq p_{i}$ and the part selected from $\lambda^{i}$, and added to give $\mu^{i}$, is at most $f\left(\lambda^{i}\right)$.

Lemma 2.6. If Proposition 2.4 (without uniqueness) is true for $a=b$, then it is true for $a=b+1$.

Proof. Suppose $\nu^{1}, \nu^{2}, \ldots, \nu^{k}$ are the partitions obtained by inserting $b$ into $\lambda^{1}, \lambda^{2}, \ldots, \lambda^{k}$ as in Proposition [2.4] To insert $b+1$ into $\lambda^{1}, \lambda^{2}, \ldots, \lambda^{k}$ we need to determine which partition $\lambda^{i}$ gets a part that is larger than it did when we inserted $b$ into $\lambda^{1}, \lambda^{2}, \ldots, \lambda^{k}$.

If the selected part of each of $\nu^{1}, \nu^{2}, \ldots, \nu^{k}$ is the first part of that partition, then we let $\mu^{2}=$ $\nu^{2}, \mu^{3}=\nu^{3}, \ldots, \mu^{k}=\nu^{k}$ and we let $\mu^{1}$ be $\nu^{1}$ except with first part one larger, i.e. $\mu_{1}^{1}=\nu_{1}^{1}+1$ and $\mu_{i}^{1}=\nu_{i}^{1}$ for $i \geq 2$. See Figure 6

Otherwise consider the smallest $i$ such that the selected part of $\nu^{i}$ is not equal to the part above it or $p_{i}$ if it is the first part of $\nu^{i}$. (Since we start by selecting the first row of $\nu^{k}$, if we have not selected the first row of every $\nu^{1}, \nu^{2}, \ldots, \nu^{k}$, there must be such an $i$.) Add 1 to this selected part in $\nu^{i}$ to obtain $\mu^{i}$. The rest of the sequence of partitions is defined by $\mu^{j}=\nu^{j}$. See Figures 7 and 8 , 


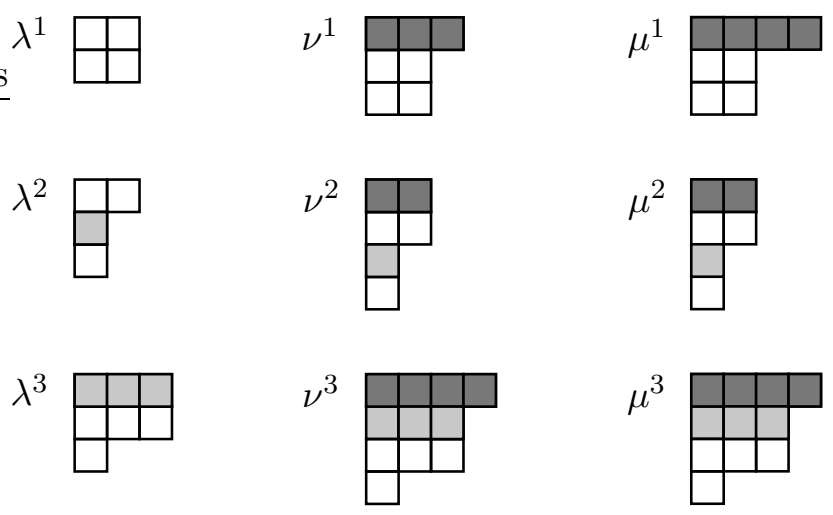

Figure 6 . Consider $\lambda^{1}, \lambda^{2}$, and $\lambda^{3}$ with $p_{2}=4$ and $p_{3}=2$ so that $A\left(\lambda^{1}, \lambda^{2}, \lambda^{3}\right)=4$. If $\nu^{1}, \nu^{2}$, and $\nu^{3}$ are obtained by inserting 9 into $\lambda^{1}, \lambda^{2}$, and $\lambda^{3}$, then $\mu^{1}, \mu^{2}$, and $\mu^{3}$ are obtained by inserting 10 into $\lambda^{1}, \lambda^{2}$, and $\lambda^{3}$.
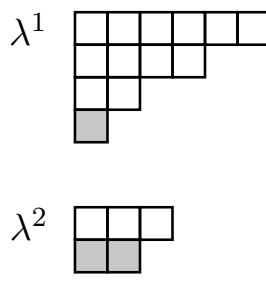

$\lambda^{3}$

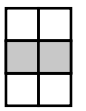

$\lambda^{4}$

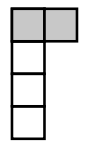

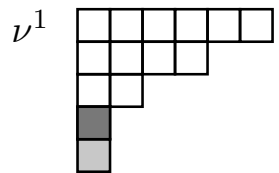

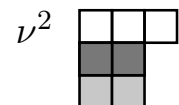

$\nu^{3}$

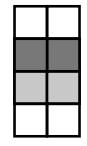

$\nu^{4}$

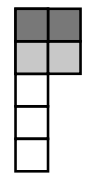

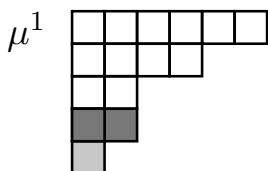

$\mu^{2}$

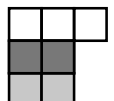

$\mu^{3}$

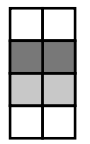

$\mu^{4}$

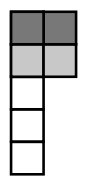

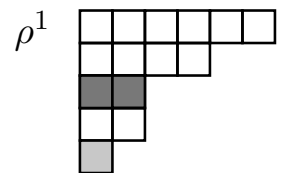

$\rho^{2}$

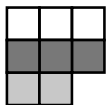

$\rho^{3}$

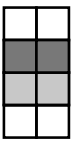

$\rho^{4}$

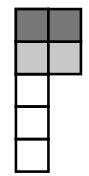

Figure 7. Consider $\lambda^{1}, \lambda^{2}, \lambda^{3}$, and $\lambda^{4}$ with $p_{2}=4, p_{3}=2$, and $p_{4}=3$. We insert 7 into $\lambda^{1}, \lambda^{2}, \lambda^{3}$, and $\lambda^{4}$ to get $\nu^{1}, \nu^{2}, \nu^{3}$, and $\nu^{4}, 8$ to get $\mu^{1}, \mu^{2}, \mu^{3}$, and $\mu^{4}$, and 9 to get $\rho^{1}, \rho^{2}, \rho^{3}$, and $\rho^{4}$.

Condition (1) follows immediately from either case since we have only added 1 to one part. Also note that we never add 1 to a row that already has length $p_{i}$ which implies condition (2).

Finally, consider the selected parts of $\mu^{1}, \mu^{2}, \ldots, \mu^{k}$. Let $i$ be as found above. For partitions $\mu^{i+1}, \ldots, \mu^{k}$ we select the same part as in $\nu^{i+1}, \ldots, \nu^{k}$. In $\mu^{i}$ we select the part to which we added 1. For partitions $\mu^{1}, \ldots, \mu^{i-1}$ we select the part directly above the selected part of $\nu^{1}, \ldots, \nu^{i-1}$ but because of our choice of $i$ these selected parts are equal to the selected parts of $\nu^{1}, \ldots, \nu^{i-1}$. Therefore selected parts have the same length as those inserted and $A\left(\nu^{1}, \nu^{2}, \ldots, \nu^{k}\right)=A\left(\mu^{1}, \mu^{2}, \ldots, \mu^{k}\right)+1$, implying condition (3).

Proof of Proposition 2.4. The two previous lemmas give the base case and inductive step necessary to prove Proposition 2.4 without the uniqueness property. All that is needed to complete the proof is to check the uniqueness of $\mu^{1}, \mu^{2}, \ldots, \mu^{k}$. 


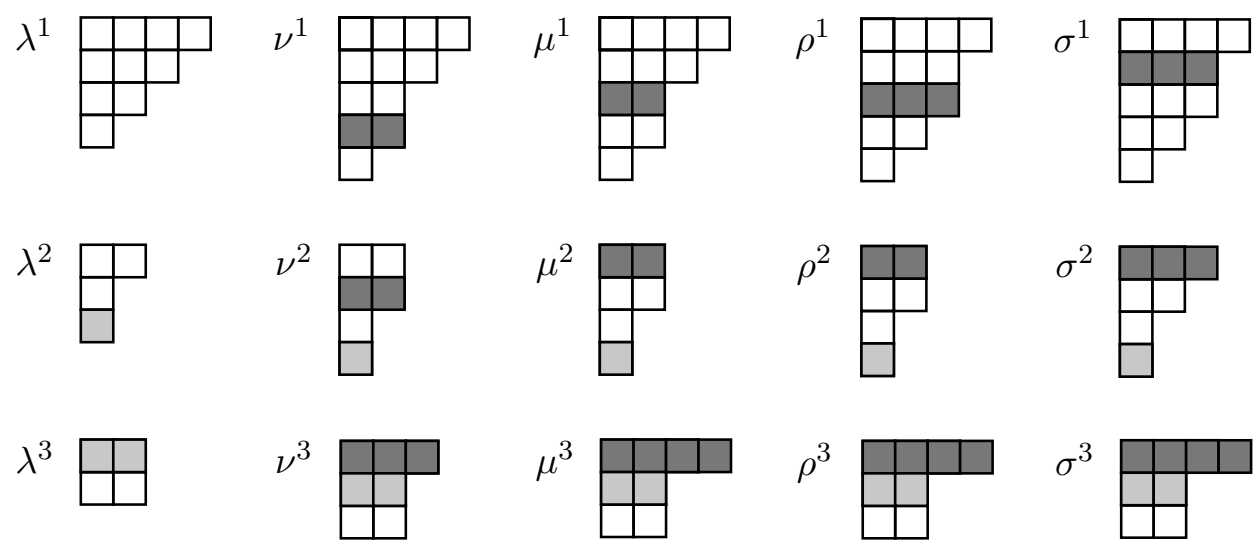

Figure 8. Consider $\lambda^{1}, \lambda^{2}$, and $\lambda^{3}$ with $p_{2}=4$ and $p_{3}=4$. We insert 7 into $\lambda^{1}$, $\lambda^{2}$, and $\lambda^{3}$ to get $\nu^{1}, \nu^{2}$, and $\nu^{3}, 8$ to get $\mu^{1}, \mu^{2}$, and $\mu^{3}, 9$ to get $\rho^{1}, \rho^{2}$, and $\rho^{3}$, and 10 to get $\sigma^{1}, \sigma^{2}$, and $\sigma^{3}$.

Suppose $\mu^{1}, \mu^{2}, \ldots, \mu^{k}$ and $\nu^{1}, \nu^{2}, \ldots, \nu^{k}$ are both sequences satisfying conditions (1), (2), and (3) of the proposition for some particular sequence $\lambda^{1}, \lambda^{2}, \ldots, \lambda^{k}$ and integer $a \geq A\left(\lambda^{1}, \lambda^{2}, \ldots, \lambda^{k}\right)$.

Since removing the selected parts of each sequence gives $\lambda^{1}, \lambda^{2}, \ldots, \lambda^{k}$, the sequences $\mu^{1}, \mu^{2}, \ldots, \mu^{k}$ and $\nu^{1}, \nu^{2}, \ldots, \nu^{k}$ must differ in a selected part. Let $i$ be the largest index so that the selected part of $\mu^{i}$ and $\nu^{i}$ are not equal. Since $i$ is the largest index where this happens, the selected parts of $\mu^{i}$ and $\nu^{i}$ must sit in the same row, say $j$. Without loss of generality, $\mu^{i}{ }_{j}>\nu^{i}{ }_{j}$.

Our selection procedure now forces the selected part of $\mu^{s}$ to be greater than or equal to the selected part of $\nu^{s}$ for $s<i$, which gives us

$$
A\left(\mu^{1}, \mu^{2}, \ldots, \mu^{k}\right)>A\left(\nu^{1}, \nu^{2}, \ldots, \nu^{k}\right) .
$$

However, both of these are equal to $a$ and so we have reached a contradiction. There cannot be a difference between the sequence of partitions $\mu^{1}, \mu^{2}, \ldots, \mu^{k}$ and the sequence of partitions $\nu^{1}, \nu^{2}, \ldots, \nu^{k}$.

This proposition will be used repeatedly to establish the bijections in sections 4 . For convenience, we establish the following notation. Let

$$
\phi_{\left\{p_{2}, \ldots, p_{k}\right\}}\left(a ; \lambda^{1}, \lambda^{2}, \ldots, \lambda^{k}\right)=\phi\left(a ; \lambda^{1}, \lambda^{2}, \ldots, \lambda^{k}\right)=\left(\mu^{1}, \mu^{2}, \ldots, \mu^{k}\right)
$$

where $\mu^{1}, \mu^{2}, \ldots, \mu^{k}$ are the partitions uniquely defined by Proposition 2.4. Of course, $\phi$ is only defined for $\lambda^{1}, \lambda^{2}, \ldots, \lambda^{k}$ and $a$ such that $A\left(\lambda^{1}, \lambda^{2}, \ldots, \lambda^{k}\right) \leq a$.

The proof of Proposition 2.4 gives us the following algorithm for insertion.

Procedure 2.7. Let $\lambda^{1}, \lambda^{2}, \ldots, \lambda^{k}$ and a be such that $A\left(\lambda^{1}, \lambda^{2}, \ldots, \lambda^{k}\right) \leq a$.

First insert a part of the same length as the part selected from $\lambda^{i}$ when calculating $A\left(\lambda^{1}, \lambda^{2}, \ldots, \lambda^{k}\right)$ to $\lambda^{i}$ to obtain $\nu^{i}$.

Now we proceed recursively, adding one square at a time to $\nu^{1}, \nu^{2}, \ldots, \nu^{k}$ until we have inserted parts whose sum is a. To add one more box to the sequence of partitions:

- If the selected part of $\nu^{1}$ is the first part, add one to this part. 
- Otherwise, find the partition $\nu^{i}$ with smallest index $i$ such that the selected part of $\nu^{i}$ is strictly less than the part above it or is strictly less than $p_{i}$ if it is the first part, and add one to this part.

When we have added a total of a boxes, let $\mu^{1}, \mu^{2}, \ldots, \mu^{k}$ be the resulting partitions. We have

$$
\phi\left(a ; \lambda^{1}, \lambda^{2}, \ldots, \lambda^{k}\right)=\left(\mu^{1}, \mu^{2}, \ldots, \mu^{k}\right) .
$$

2.3. Relationship between selection and insertion. The last line of Proposition 2.4 also shows that insertion is reversible. Since the rows added by $\phi$ are those selected when calculating $A\left(\mu^{1}, \mu^{2}, \ldots, \mu^{k}\right)$ and $a=A\left(\mu^{1}, \mu^{2}, \ldots, \mu^{k}\right)$, we can undo insertion by using selection and removal. It will be useful to formally note this consequence of Proposition 2.4

Corollary 2.8. Let $p_{2}, p_{3}, \ldots, p_{k}$ be integers.

(1) For any sequence of $k$ partitions $\lambda^{1}, \lambda^{2}, \ldots, \lambda^{k}$ such that

$$
f\left(\lambda^{2}\right) \leq p_{2}, f\left(\lambda^{3}\right) \leq p_{3}, \ldots, f\left(\lambda^{k}\right) \leq p_{k}
$$

and integer a such that $a \geq A\left(\lambda^{1}, \lambda^{2}, \ldots, \lambda^{k} ; p_{2}, \ldots, p_{k}\right)$ we have

$$
\psi\left(\phi\left(a ; \lambda^{1}, \lambda^{2}, \ldots, \lambda^{k}\right)\right)=\left(a ; \lambda^{1}, \lambda^{2}, \ldots, \lambda^{k}\right) .
$$

(2) For any sequence of $k$ partitions $\mu^{1}, \mu^{2}, \ldots, \mu^{k}$ such that

$$
f\left(\mu^{2}\right) \leq p_{2}, f\left(\mu^{3}\right) \leq p_{3}, \ldots, f\left(\mu^{k}\right) \leq p_{k}
$$

we have

$$
\phi\left(\psi\left(\mu^{1}, \mu^{2}, \ldots, \mu^{k}\right)\right)=\left(\mu^{1}, \mu^{2}, \ldots, \mu^{k}\right) .
$$

2.4. Iterative removal of selected parts. As a final remark, we note that if $\psi_{2}\left(\mu^{1}, \mu^{2}, \ldots, \mu^{k}\right)=$ $\left(\lambda^{1}, \lambda^{2}, \ldots, \lambda^{k}\right)$, then

$$
f\left(\lambda^{2}\right) \leq p_{2}, f\left(\lambda^{3}\right) \leq p_{3}, \ldots, f\left(\lambda^{k}\right) \leq p_{k} .
$$

Therefore we can apply $\psi_{1}$ or $\psi_{2}$ to $\lambda^{1}, \lambda^{2}, \ldots, \lambda^{k}$ and in general we can reapply $\psi_{2}$, the removal of selected parts, any number of times. The following lemma describes more precisely what happens to selected parts when $\psi_{2}$ is applied more than once. See Figure 9 .

Lemma 2.9. For any sequence of $k$ partitions $\mu^{1}, \mu^{2}, \ldots, \mu^{k}$ such that

$$
f\left(\mu^{2}\right) \leq p_{2}, f\left(\mu^{3}\right) \leq p_{3}, \ldots, f\left(\mu^{k}\right) \leq p_{k}
$$

the selected rows of $\psi_{2}\left(\mu^{1}, \mu^{2}, \ldots, \mu^{k}\right)$ are rows that sit strictly below the selected rows of $\mu^{1}, \mu^{2}, \ldots, \mu^{k}$ in $\mu^{1}, \mu^{2}, \ldots, \mu^{k}$.

In particular we have

$$
A\left(\psi_{2}\left(\mu^{1}, \mu^{2}, \ldots, \mu^{k}\right) ; p_{2}, p_{3}, \ldots, p_{k}\right) \leq A\left(\mu^{1}, \mu^{2}, \ldots, \mu^{k} ; p_{2}, p_{3}, \ldots, p_{k}\right) .
$$

Proof. This follows by a simple inductive argument.

Let $\psi_{2}\left(\mu^{1}, \mu^{2}, \ldots, \mu^{k}\right)=\left(\nu^{1}, \nu^{2}, \ldots, \nu^{k}\right)$. In both $\mu^{k}$ and $\nu^{k}$ we select the first part. However, the first part of $\nu^{k}$ is the second part of $\mu^{k}$ and so the result holds for $\mu^{k}$ and $\nu^{k}$.

Moreover if the result is true for $\mu^{i}$ and $\nu^{i}$, and if we selected the $h$ th row of $\mu^{i}$ and the $j$ th row of $\nu^{i}$, then we have $h \leq j$. This implies $\mu^{i}{ }_{h} \geq \nu^{i}{ }_{j}$. Then the selected rows of $\mu^{i-1}$ and $\nu^{i-1}$ are $h+\left(p_{i}-\mu_{h}^{i}\right)$ and $j+\left(p_{i}-\nu^{i}{ }_{j}\right)$ respectively and $h+\left(p_{i}-\mu_{h}^{i}\right) \leq j+\left(p_{i}-\nu^{i}{ }_{j}\right)$ as desired.

The procedures presented here are the main tools needed build the combinatorial proof of (2). These procedures can also be used to obtain other bijections as is shown in Appendix A of [7]. 

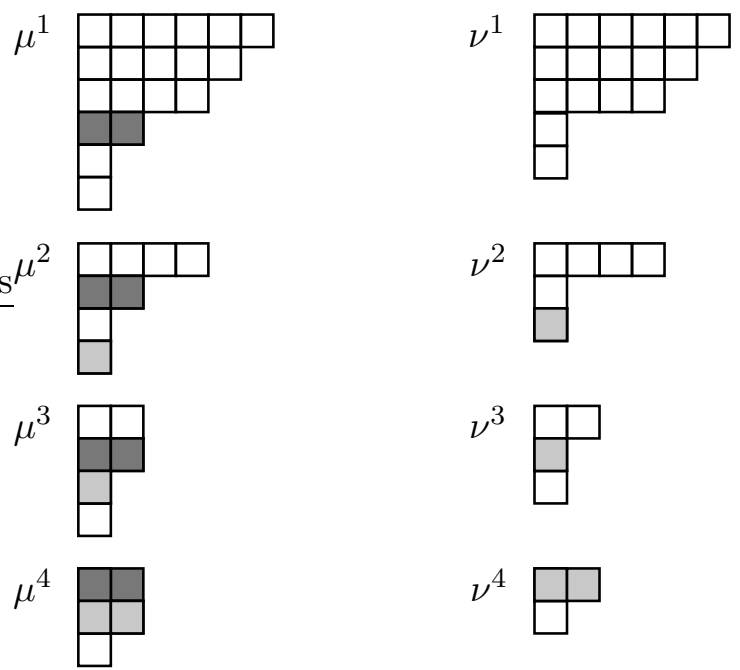

Figure 9. For $p_{2}=4, p_{3}=2$, and $p_{4}=3$, we see that for $\psi_{2}\left(\mu^{1}, \mu^{2}, \mu^{3}, \mu^{4}\right)=$ $\left(\nu^{1}, \nu^{2}, \nu^{3}, \nu^{4}\right)$. Also note that $A\left(\mu^{1}, \mu^{2}, \mu^{3}, \mu^{4}\right)=8 \geq A\left(\nu^{1}, \nu^{2}, \nu^{3}, \nu^{4}\right)=4$.

\section{Definition of $(k, m)$-RAnK}

In this section, we will define notion of $(k, m)$-rank for partitions with at least $k$ successive $m$-Durfee rectangles. (This generalizes the $(2, m)$-rank for partitions with at least two successive $m$-Durfee rectangles found in $[\underline{8}$.)

First, given a partition $\lambda$ with $k$ successive $m$-Durfee rectangles, denote by $\lambda^{i}$ the partition to the right of the $i$ th $m$-Durfee rectangle and denote by $\alpha$ the partition below the $k$ th $m$-Durfee rectangle. Moreover, let $N_{1}, N_{2}, \ldots, N_{k}$ denote the widths of the first $k$ successive $m$-Durfee rectangles. Note that, for all $i, \lambda^{i}$ has at most $N_{i}+m$ parts and, for $i \geq 2$, the largest part of $\lambda^{i}$ is at most $N_{i-1}-N_{i}$. See Figure 10
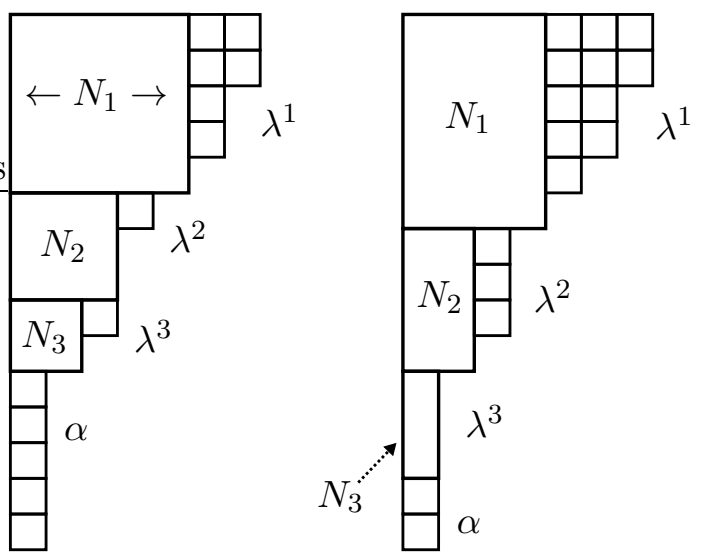

Figure 10. Successive Durfee rectangles of width $N_{1}, N_{2}$, and $N_{3}$ and names for the partitions to the right, $\lambda^{1}, \lambda^{2}$, and $\lambda^{3}$, and below, $\alpha$, those Durfee rectangles.

Dyson's original definition of rank applies to a partition with (at least) one Durfee square and compares the largest part of the $\lambda^{1}$ to the number of parts of $\alpha$. Our $(k, m)$-rank will compare parts to the right of the $k$ successive $m$-Durfee rectangles to the number of parts of $\alpha$. 
Let $p_{i}=N_{i-1}-N_{i}$. Then, as we noted above, we have $f\left(\lambda^{i}\right) \leq p_{i}$ for $i \geq 2$. Therefore, we may apply selection to the sequence of partitions to the right of our Durfee rectangles, $\lambda^{1}, \lambda^{2}, \ldots, \lambda^{k}$.

See Figure 11 for two examples of this selection process. On the left hand side, we consider $\lambda$ with 3 successive Durfee squares. First we select the first part of $\lambda^{3}$. Next we select the $1+(1-1)=1$ st part of $\lambda^{2}$ and we select the $1+(2-1)=2$ nd part of $\lambda^{1}$. On the right hand side, we consider $\lambda$ with 3 successive 1-Durfee rectangles. First we select the first part of $\lambda^{3}$, then we select the $1+(1-0)=2$ nd part of $\lambda^{2}$ and finally the $2+(2-1)=3$ rd part of $\lambda^{1}$.
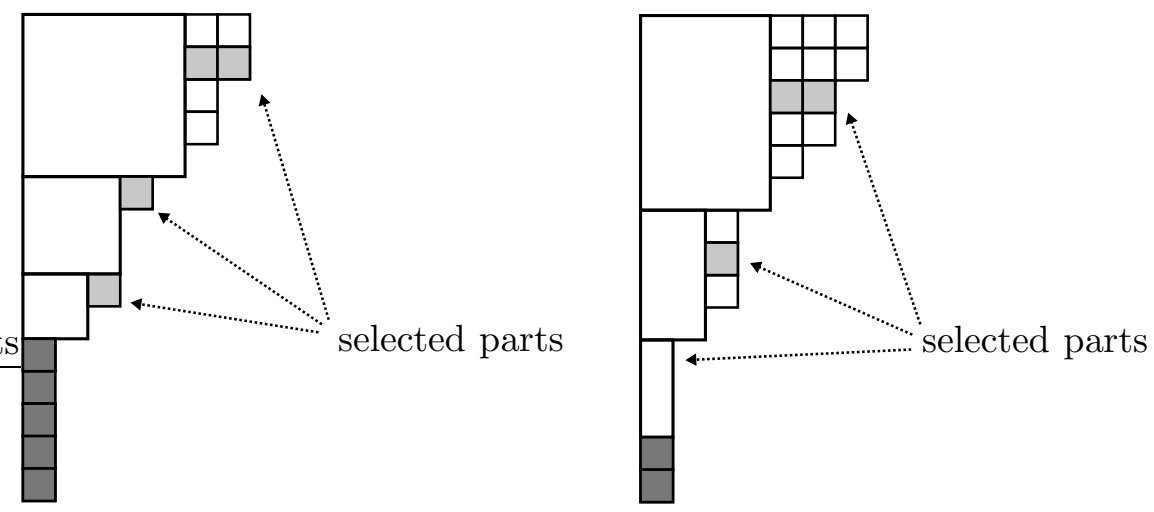

Figure 11. For partition $\lambda=(7,7,6,6,5,4,3,3,3,2,1,1,1,1,1)$, we have $a_{3,0}(\lambda)=$ $2+1+1=4, b_{3,0}(\lambda)=5$, and $r_{3,0}(\lambda)=4-5=-1$, while $a_{3,1}(\lambda)=2+1+0=3$, $b_{3,1}(\lambda)=2$, and $r_{3,1}(\lambda)=3-2=1$.

Notice that in these examples, the selected part of the partition $\lambda^{i}$ is never below the bottom row of the $m$-Durfee rectangle sitting to its left. This is true in general as stated by the following lemma.

Lemma 3.1. If the $j$ th part of $\lambda^{i}$ has been selected, then $j \leq N_{i}+m$.

Proof. We will prove the stronger statement that if the $j$ th part of $\lambda^{i}$ has been selected then $j \leq 1+N_{i}-N_{k}$.

If $m \leq 0$, the $k$ th successive $m$-Durfee square has non-zero height, and so its width is $N_{k} \geq 1-m$, which gives us

$$
1+N_{i}-N_{k} \leq N_{i}+m
$$

If $m>0$, we get $N_{k} \geq 0$ so

$$
1+N_{i}-N_{k} \leq N_{i}+1 \leq N_{i}+m .
$$

Therefore the statement in the lemma follows from $j \leq 1+N_{i}-N_{k}$.

To show that $j \leq 1+N_{i}-N_{k}$, we proceed by induction, starting with $\lambda^{k}$ and moving up to $\lambda^{1}$.

We select the first row of $\lambda^{k}$ and have $1=1+N_{k}-N_{k}$.

If the $j$ th row of $\lambda^{i}$ has been selected, we select the $j+p_{i}-\lambda_{j}^{i}=\left(j+\left(N_{i-1}-N_{i}\right)-\lambda^{i}{ }_{j}\right)$ th row of $\lambda^{i-1}$. Now our inductive hypothesis says that then $j \leq 1+N_{i}-N_{k}$. Hence we see that

$$
\begin{aligned}
j+\left(N_{i-1}-N_{i}\right)-\lambda^{i}{ }_{j} & \leq 1+N_{i}-N_{k}+N_{i-1}-N_{i}-\lambda^{i}{ }_{j} \\
& \leq 1+N_{i-1}-N_{k}
\end{aligned}
$$

as desired.

Finally, we can give the definition of $(k, m)$-rank, $r_{k, m}(\lambda)$. 
Definition 3.2. For $k>0$, consider a partition $\lambda$ with $k$ successive $m$-Durfee rectangles of width $N_{1}, N_{2}, \ldots, N_{k}$. Let $p_{i}=N_{i-1}-N_{i}$. Also, let $\lambda^{1}, \lambda^{2}, \ldots, \lambda^{k}$ be the partitions to the right of the Durfee rectangles and let $\alpha$ be the partition below the $k$ th Durfee rectangle. Define

- $a_{k, m}(\lambda)=A\left(\lambda^{1}, \lambda^{2}, \ldots, \lambda^{k} ; p_{2}, p_{3}, . ., p_{k}\right)$, the sum of the parts selected from $\lambda^{1}, \lambda^{2}, \ldots, \lambda^{k}$,

- $b_{k, m}(\lambda)=\ell(\alpha)$, the number of parts of $\alpha$ and

- $r_{k, m}(\lambda)=a_{k, m}(\lambda)-b_{k, m}(\lambda)$.

In words, our definition of $(k, m)$-rank selects parts to the right of the $k$ successive $m$-Durfee rectangles of $\lambda$ and compares the total size of these parts to the number of parts below the Durfee rectangles. In the case $k=1$ and $m=0$ this corresponds exactly to Dyson's original definition. See Figure 11 for examples.

\section{SyMmetries}

Let $h(n, k, m, r)$ be the number of partitions of $n$ with $(k, m)$-rank equal to $r$. Similarly, let $h(n, k, m, \leq r)$ be the number of partitions of $n$ with $(k, m)$-rank less than or equal to $r$ and let $h(n, k, m, \geq r)$ be the number of partitions of $n$ with $(k, m)$-rank greater than or equal to $r$.

There are relationships between these numbers that generalize the symmetries used in Dyson's proof of Euler's pentagonal number theorem. These relationships will be proved in a completely combinatorial way and, in the following section, they will be used to establish the generalized Schur identities by simple algebraic manipulation.

Recall that $q_{k-1}(n)$ denotes the number of partitions with at most $k-1$ Durfee squares, so that $p(n)-q_{k-1}(n)$ is the number of partitions with at least $k$ Durfee squares.

The following two observations follow directly from our definitions since $(k, 0)$-rank is only defined for the set of partitions with $k$ non-empty Durfee squares, whereas when $m>0,(k, m)$-rank is defined for the set of all partitions.

Unless otherwise stated we implicitly assume that $n, k, m, r \in \mathbb{Z}$ and $k>0$.

Observation 4.1 (First observation). For $m=0$,

$$
h(n, k, 0, \leq r)+h(n, k, 0, \geq r+1)=p(n)-q_{k-1}(n) .
$$

Observation 4.2 (Second observation). For $m>0$,

$$
h(n, k, m, \leq r)+h(n, k, m, \geq r+1)=p(n) .
$$

There are also more complicated relations between these numbers.

Theorem 4.3 (First symmetry). For any $r \in \mathbb{Z}$ and $m=0$,

$$
h(n, k, 0, r)=h(n, k, 0,-r) .
$$

Theorem 4.4 (Second symmetry). For $m, r \in \mathbb{Z}$, if $r>0$ or if $m \leq 0$,

$$
h(n, k, m, \leq-r)=h(n-r-k(m+1), k, m+2, \geq-r) .
$$

These symmetries generalize the symmetries in Dyson's proof and will be proved bijectively. The first will be proved by a bijection generalizing conjugation and the second by a bijection generalizing $d_{r}$, Dyson's map for his new symmetry. 
4.1. First symmetry. To prove the first symmetry we are looking for a conjugation-like bijection that takes as its input a partition with $(k, 0)$-rank $r$ and outputs a partition with $(k, 0)$-rank $-r$. The following theorem says that this bijection will not change the Durfee square structure of the partition and suggests that we find a way to simply exchange the selected parts of $\lambda^{1}, \ldots, \lambda^{k}$, the partitions to the right of the Durfee squares, with the first column of $\alpha$, the partition below the Durfee squares.

Theorem 4.5. For any integers $s, t \geq 0$, the number of partitions $\lambda$ of $n$ with $k$ successive Durfee squares of widths $N_{1}, N_{2}, \ldots, N_{k}$ such that $a_{k, 0}(\lambda)=s$ and $b_{k, 0}(\lambda)=t$ is equal to the number of partitions $\mu$ of $n$ with $k$ successive Durfee squares of widths $N_{1}, N_{2}, \ldots, N_{k}$ such that $a_{k, 0}(\mu)=t$ and $b_{k, 0}(\mu)=s$.

The following two corollaries follow immediately from this theorem.

Corollary 4.6. For any $r \in \mathbb{Z}$, the number of partitions $\lambda$ of $n$ with $k$ successive Durfee squares of widths $N_{1}, N_{2}, \ldots, N_{k}$ such that $r_{k, 0}(\lambda)=r$ is equal to the number of partitions $\mu$ of $n$ with $k$ successive Durfee squares of widths $N_{1}, N_{2}, \ldots, N_{k}$ such that $r_{k, 0}(\mu)=-r$.

Corollary 4.7. For any $r \in \mathbb{Z}$, the number of partitions $\lambda$ of $n$ with $k$ successive Durfee squares and $r_{k, 0}(\lambda)=r$ is equal to the number of partitions $\mu$ of $n$ with $k$ successive Durfee squares and $r_{k, 0}(\mu)=-r$.

Note that Corollary 4.7 is exactly Theorem 4.3, the first symmetry.

To prove Theorem 4.5 and its corollaries, we present a map,

$$
\mathfrak{C}^{k}: \mathcal{P} \backslash \mathcal{Q}_{k-1} \rightarrow \mathcal{P} \backslash \mathcal{Q}_{k-1}
$$

Recall that

$$
\mathcal{Q}_{k-1}=\{\text { partitions with at most } k-1 \text { Durfee squares }\}
$$

and so

$$
\mathcal{P} \backslash \mathcal{Q}_{k-1}=\{\text { partitions with at least } k \text { Durfee squares }\}
$$

Procedure 4.8 (Generalized Conjugation). Let $\lambda$ be a partition with at least $k$ Durfee squares.

Let $\alpha$ be the partition below the $k$ th Durfee square of $\lambda$ and $\lambda^{1}, \lambda^{2}, \ldots, \lambda^{k}$ be the partitions to the right of these squares.

Let $N_{1}, N_{2}, \ldots, N_{k}$ be the size of the $k$ successive Durfee squares and let

$$
p_{2}=N_{1}-N_{2}, p_{3}=N_{2}-N_{3}, \ldots, p_{k}=N_{k-1}-N_{k}
$$

We iteratively remove selected parts from $\lambda^{1}, \lambda^{2}, \ldots, \lambda^{k}$ by using $\psi_{2} N_{k}$ times to obtain:

$$
\psi_{2}{ }^{N_{k}}\left(\lambda^{1}, \lambda^{2}, \ldots, \lambda^{k}\right)=\left(\nu^{1}, \nu^{2}, \ldots, \nu^{k}\right)
$$


As these rows are removed, we record the sum of the parts removed at each step. We record this information in a partition $\beta$ as follows. The partition $\beta$ is defined by giving its conjugate.

$$
\begin{gathered}
\beta_{1}^{\prime}=\psi_{1}\left(\lambda^{1}, \lambda^{2}, \ldots, \lambda^{k}\right), \\
\beta_{2}^{\prime}=\psi_{1}\left(\psi_{2}\left(\lambda^{1}, \lambda^{2}, \ldots, \lambda^{k}\right)\right), \\
\vdots \\
\beta_{N_{k}-1}^{\prime}=\psi_{1}\left(\psi_{2}^{N_{k}-2}\left(\lambda^{1}, \lambda^{2}, \ldots, \lambda^{k}\right)\right), \\
\beta_{N_{k}}^{\prime}=\psi_{1}\left(\psi_{2}^{N_{k}-1}\left(\lambda^{1}, \lambda^{2}, \ldots, \lambda^{k}\right)\right) .
\end{gathered}
$$

Next we insert $\alpha_{N_{k}}^{\prime}, \alpha_{N_{k}-1}^{\prime}, \ldots, \alpha_{1}^{\prime}$ into $\nu^{1}, \nu^{2}, \ldots, \nu^{k}$ in that order giving us

$$
\phi\left(\alpha_{1}^{\prime} ; \phi\left(\alpha_{2}^{\prime} ; \ldots \phi\left(\alpha_{N_{k}-1}^{\prime} ; \phi\left(\alpha_{N_{k}}^{\prime} ; \nu^{1}, \ldots, \nu^{k}\right)\right) \ldots\right)\right)=\left(\mu^{1}, \ldots, \mu^{k}\right) .
$$

Let $\mu$ be a new partition defined by having

- $k$ successive Durfee squares of widths $N_{1}, N_{2}, \ldots, N_{k}$,

- $\mu^{1}, \mu^{2} \ldots, \mu^{k}$ to the right of these squares, and

- $\beta$ below the kth Durfee square.

Then $\mathfrak{C}^{k}(\lambda)=\mu$.

We will also write $\mathfrak{C}$ for $\mathfrak{C}^{k}$.

The map $\mathfrak{C}$ consists of using the tools from Section 2 to first remove selected parts from the partitions to the right of the Durfee squares $N_{k}$ times and record the sum of the removed parts as the $N_{k}$ columns of the partition $\beta$ (to be place below the Durfee squares). Second, we insert the $N_{k}$ columns of the partition $\alpha$ (that was below the Durfee squares) into the partitions to the right of the Durfee squares (from which we just removed parts). As such we are exchanging some parts to the right of the Durfee squares with the parts below the Durfee squares.

Because of the way the maps $\phi$ and $\psi$ are defined, this exchange of parts is well-defined and is an involution. Before proving this we will give two examples of applications of $\mathfrak{C}$. These are found in figures 12 and 13 .

Proof of Theorem 4.5. Let $\lambda$ be a partition with

$$
\begin{aligned}
a_{k, m}(\lambda) & =s \text { and } \\
b_{k, m}(\lambda) & =t .
\end{aligned}
$$

To prove Theorem 4.5 we will show that $\mathfrak{C}$ is an involution that exchanges $a_{k, m}$ and $b_{k, m}$ while preserving the Durfee square structure of $\lambda$.

First we want to show that $\mathfrak{C}$ is well-defined.

For each $2 \leq i \leq k, \lambda^{i}$ fits to the right of the $i$ th Durfee rectangle and below the $(i-1)$ st Durfee rectangle. As a consequence, its largest part satisfies $f\left(\lambda^{i}\right) \leq N_{i}-N_{i-1}=p_{i}$.

Therefore, we may select parts from and apply the maps $\psi_{1}$ and $\psi_{2}$ to $\lambda^{1}, \lambda^{2}, \ldots, \lambda^{k}$. Moreover, as we remarked in of Section 2.4, the iterated applications we do here are also fine. 
$\lambda$

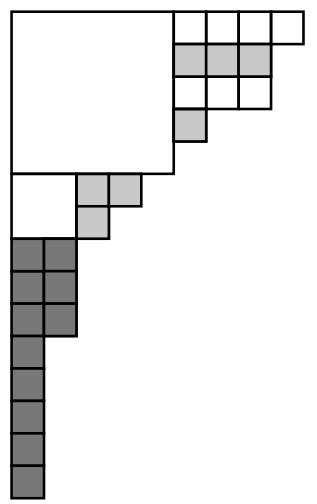

$\nu^{1}$

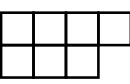

$\nu^{2}$

$\alpha^{\prime}$

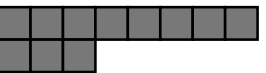

$\beta^{\prime}$

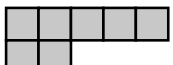

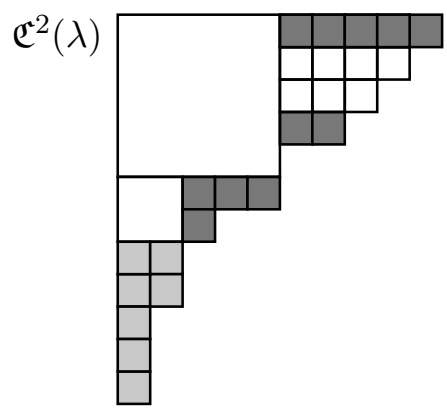

Figure 12. Applying $\mathfrak{C}^{2}$ to the partition $\lambda=(9,8,8,6,5,4,3,2,2,2,1,1,1,1,1)$ gives $\mathfrak{C}^{2}(\lambda)=(10,9,8,7,5,5,3,2,2,1,1,1)$. Intermediate steps are $\nu^{1}, \nu^{2}, \alpha^{\prime}$, and $\beta^{\prime}$ as shown.
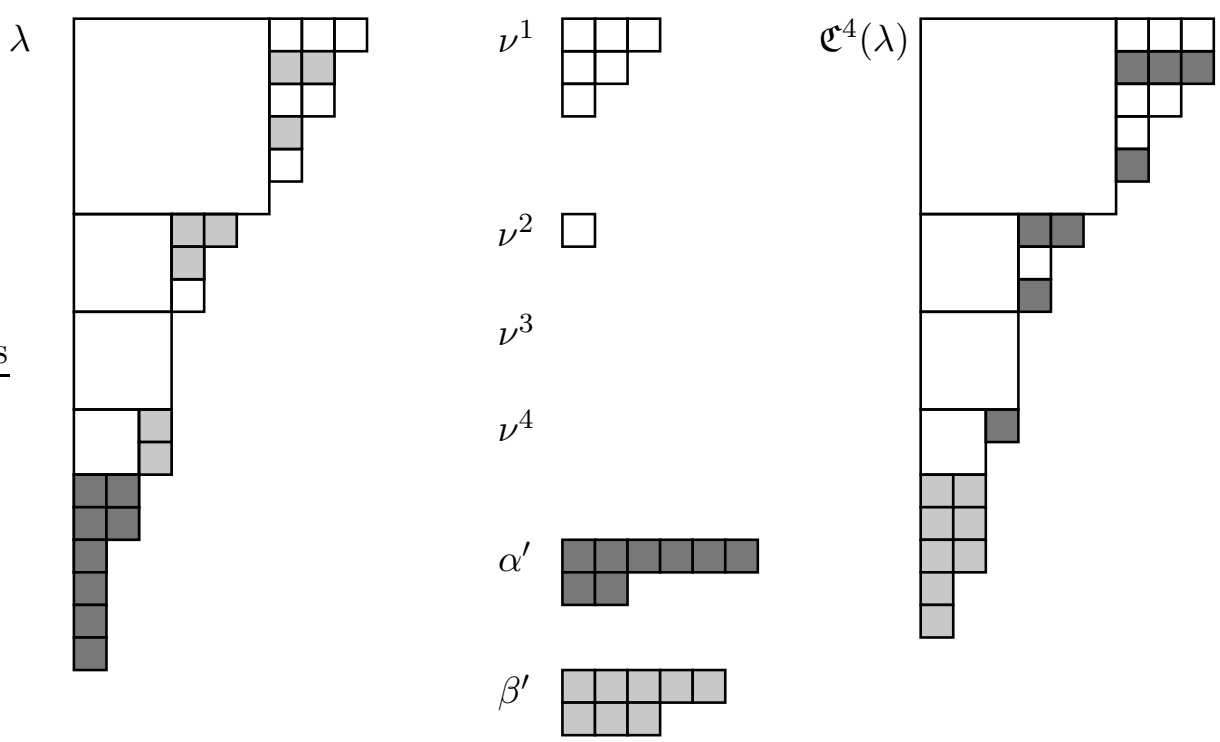

Figure 13. Applying $\mathfrak{C}^{4}$ to $\lambda=(9,8,8,7,7,6,5,4,4,3,3,3,3,3,2,2,1,1,1,1)$ gives $\mathfrak{C}^{4}(\lambda)=(9,9,8,7,7,6,5,4,4,3,3,3,3,2,2,2,2,1,1)$. Intermediate steps are $\nu^{1}, \nu^{2}, \nu^{3}, \nu^{4}, \alpha^{\prime}$, and $\beta^{\prime}$ as shown.

Next we want to check that $\beta$ is a partition and that $\beta$ fits belo the $k$ th Durfee square. Lemma 2.9 tells us that

$$
\psi_{1}\left(\psi_{2}\left(\mu^{1}, \mu^{2}, \ldots, \mu^{k}\right)\right) \leq \psi_{1}\left(\mu^{1}, \mu^{2}, \ldots, \mu^{k}\right)
$$

for any $\mu^{1}, \mu^{2}, \ldots, \mu^{k}$. Therefore,

$$
\psi_{1}\left(\psi_{2}^{j}\left(\lambda^{1}, \lambda^{2}, \ldots, \lambda^{k}\right)\right) \leq \psi_{1}\left(\psi_{2}^{j-1}\left(\lambda^{1}, \lambda^{2}, \ldots, \lambda^{k}\right)\right)
$$

for all $j \geq 1$. In other words, $\beta_{j+1}^{\prime} \leq \beta_{j}^{\prime}$. Since $\beta_{1}^{\prime} \geq \beta_{2}^{\prime} \geq \ldots \geq \beta_{N_{k}}^{\prime}$, we see that $\beta$ a partition. Also $\beta^{\prime}$ has at most $N_{k}$ parts which implies that $f(\beta) \leq N_{k}$. Therefore, we can place $\beta$ below the $k$ th Durfee square whose size is $N_{k}$.

We must also show that $\alpha^{\prime}$ can be inserted into the partitions to the right of the Durfee squares. Consider

$$
\psi_{2}^{N_{k}}\left(\lambda^{1}, \lambda^{2}, \ldots, \lambda^{k}\right)=\left(\nu^{1}, \nu^{2}, \ldots, \nu^{k}\right)
$$


Since we have simply removed parts, we have

$$
f\left(\nu^{2}\right) \leq p_{2}, f\left(\nu^{3}\right) \leq p_{3}, \ldots, f\left(\nu^{k}\right) \leq p_{k} .
$$

Moreover, for $1 \leq i \leq k, \nu^{i}$ has at most $N_{i}-N_{k}$ parts. (In particular, $\nu^{k}$ is empty.) This means that when we select parts from $\nu^{1}, \nu^{2}, \ldots, \nu^{k}$, we select the $\left(1+N_{i}-N_{k}\right)$ th part of $\nu^{i}$ which is always empty. As a consequence, $A\left(\nu^{1}, \nu^{2}, \ldots, \nu^{k} ; p_{2}, p_{3}, \ldots, p_{k}\right)=0$.

Therefore we can insert $\alpha^{\prime}{ }_{N_{k}} \geq 0$ into $\nu^{1}, \nu^{2}, . ., \nu^{k}$. As well, since $A\left(\phi\left(\alpha^{\prime}{ }_{i} ; \ldots\right)\right)=\alpha^{\prime}{ }_{i}$ and $\alpha^{\prime}{ }_{i} \leq \alpha^{\prime}{ }_{i-1}$ we can insert $\alpha^{\prime}{ }_{i-1}$ into $\phi\left(\alpha_{i}^{\prime} ; \ldots \phi\left(\alpha_{N_{k-1}}^{\prime} ; \phi\left(\alpha_{N_{k}}^{\prime} ; \nu^{1}, \ldots, \nu^{k}\right)\right) \ldots\right)$.

Finally, each of these insertions adds at most one part to each partition and does not give partitions whose largest parts are greater than $p_{i}$. Therefore

$$
f\left(\mu^{2}\right) \leq p_{2}, f\left(\mu^{3}\right) \leq p_{3}, \ldots, f\left(\mu^{k}\right) \leq p_{k}
$$

and, for $1 \leq i \leq k, \mu^{i}$ has at most $\left(N_{i}-N_{k}\right)+N_{k}=N_{i}$ parts. Each $\mu^{1}, \mu^{2}, \ldots, \mu^{k}$ can be inserted to the right of each of the first $k$ Durfee squares and this shows that $\mathfrak{C}$ is well-defined.

To see that $\mathfrak{C}$ is an involution, we simply use the relationship between $\phi$ and $\psi$ summarized in Corollary 2.8. Say $\mathfrak{C}(\lambda)=\mu$ with $\alpha, \beta,\left(\lambda^{1}, \lambda^{2}, \ldots, \lambda^{k}\right)$, and $\left(\mu^{1}, \mu^{2}, \ldots, \mu^{k}\right)$ as in the definition of Procedure 4.8. We will apply $\mathfrak{C}$ to $\mu$.

Applying $\psi$ undoes the insertions done by $\phi$, and we get

$$
\begin{aligned}
\psi_{1}\left(\mu^{1}, \mu^{2}, \ldots, \mu^{k}\right) & =\psi_{1}\left(\phi\left(\alpha_{1}^{\prime} ; \ldots\right)\right)=\alpha_{1}^{\prime} \text { and } \\
\psi_{1}\left(\psi_{2}\left(\mu^{1}, \mu^{2}, \ldots, \mu^{k}\right)\right) & =\psi_{1}\left(\psi_{2}\left(\phi\left(\alpha_{1}^{\prime} ; \phi\left(\alpha_{2}^{\prime} ; \ldots\right)\right)\right)\right)=\alpha_{2}^{\prime} .
\end{aligned}
$$

Similarly,

$$
\begin{gathered}
\psi_{1}\left(\psi_{2}^{2}\left(\mu^{1}, \mu^{2}, \ldots, \mu^{k}\right)\right)=\alpha_{3}^{\prime}, \\
\vdots \\
\psi_{1}\left(\psi_{2}{ }^{N_{k}-2}\left(\mu^{1}, \mu^{2}, \ldots, \mu^{k}\right)\right)=\alpha_{N_{k}-1}^{\prime}, \\
\psi_{1}\left(\psi_{2}{ }^{N_{k}-1}\left(\mu^{1}, \mu^{2}, \ldots, \mu^{k}\right)\right)=\alpha_{N_{k}}^{\prime},
\end{gathered}
$$

and

$$
\left.\psi_{2}^{N_{k}}\left(\mu^{1}, \mu^{2}, \ldots, \mu^{k}\right)\right)=\left(\nu^{1}, \nu^{2}, . ., \nu^{k}\right) .
$$

Next we insert $\beta_{N_{k}}^{\prime}, \beta_{N_{k}-1}^{\prime}, \ldots, \beta_{1}^{\prime}$ into $\nu^{1}, \nu^{2}, \ldots, \nu^{k}$. Since these are the parts originally removed by $\psi$ from $\lambda^{1}, \lambda^{2}, \ldots, \lambda^{k}$ to give $\nu^{1}, \nu^{2}, . ., \nu^{k}$, we have

$$
\phi\left(\beta_{1}^{\prime} ; \phi\left(\beta_{2}^{\prime} ; \ldots \phi\left(\beta_{N_{k}-1}^{\prime} ; \phi\left(\beta_{N_{k}}^{\prime} ; \nu^{1}, \ldots, \nu^{k}\right)\right) \ldots\right)\right)=\left(\lambda^{1}, \lambda^{2}, \ldots, \lambda^{k}\right) .
$$

Since $\alpha$ goes below the $k$ th Durfee square and $\lambda^{1}, \lambda^{2}, \ldots, \lambda^{k}$ to the right of the Durfee squares, we get $\mathfrak{C}(\mu)=\lambda$. A second application of $\mathfrak{C}$ undoes the first application and indeed $\mathfrak{C}$ is an involution.

Finally, we note that

$$
a_{k, m}(\mu)=A\left(\mu^{1}, \mu^{2}, \ldots, \mu^{k} ; p_{2}, p_{3}, \ldots, p_{k}\right)=\psi_{1}\left(\mu^{1}, \mu^{2}, \ldots, \mu^{k}\right)=\alpha_{1}^{\prime}=b_{k, m}(\lambda)=t
$$

and since $\mathfrak{C}$ is an involution $b_{k, m}(\mu)=a_{k, m}(\lambda)=s$. 
4.2. Second Symmetry. The second symmetry will follow from the following theorem. It will be proved by giving a bijection that uses insertion to generalize Dyson's map.

Theorem 4.9. For any $m, r, t \in \mathbb{Z}$ such that $t \geq 0$, the number of partitions $\lambda$ of $n$ with $k$ successive $m$-Durfee rectangles, of non-zero widths $N_{1}, N_{2}, \ldots, N_{k}$, with $b_{k, m}(\lambda)=t$ and $r_{k, m}(\lambda) \leq-r$ is equal to the number of partitions $\mu$ of $n-r-k(m+1)$ with $k$ successive $(m+2)$-Durfee rectangles, of widths $N_{1}-1, N_{2}-1, \ldots, N_{k}-1$, with $a_{k, m+2}(\mu)=t-r$ and $b_{k, m+2}(\mu) \leq t$.

Before proving our theorem, note that the following two corollaries follow immediately.

Corollary 4.10. For any $m, r \in \mathbb{Z}$, the number of partitions $\lambda$ of $n$ with $k$ successive $m$-Durfee rectangles, of non-zero widths $N_{1}, N_{2}, \ldots, N_{k}$, with $r_{k, m}(\lambda) \leq-r$ is equal to the number of partitions $\mu$ of $n-r-k(m+1)$ with $k$ successive $(m+2)$-Durfee rectangles, of widths $N_{1}-1, N_{2}-1, \ldots$, $N_{k}-1$, with $r_{k, m+2}(\mu) \geq-r$.

In Theorem 4.9] and Corollary 4.10, one side of the identity requires non-zero width Durfee rectangles while on the other side zero width Durfee rectangles are allowed. (Durfee rectangles of height zero are never allowed as stated in the definition of Durfee rectangles.) There are two situations in which the widths of the $k$ successive $m$-Durfee rectangles are forced to be non-zero.

First, since we require $m$-Durfee rectangles to have non-zero height, the width of the rectangles is at least $1-m$. When $m \leq 0,1-m>0$ and so the width if forced to be non-zero.

Second, if $r_{k, m}(\lambda) \leq-r$, then we must have $b_{k, m}(\lambda) \geq r$. If in addition $r>0$, then $b_{k, m}(\lambda)>0$. Since $b_{k, m}(\lambda)$ is the size of the first column of $\alpha$ the partition which sits below the $k$ th successive $m$-Durfee rectangle, notice that this $m$-Durfee rectangle must have non-zero width. Therefore if $r_{k, m}(\lambda) \leq-r$ and $r>0$, all $m$-Durfee rectangles of $\lambda$ have non-zero width.

These cases give the following corollary which is Theorem 4.4

Corollary 4.11. For any $m, r \in \mathbb{Z}$ such that $m \leq 0$ or $r>0$, the number of partitions $\lambda$ of $n$ with $r_{k, m}(\lambda) \leq-r$ is equal to the number of partitions $\mu$ of $n-r-k(m+1)$ with $r_{k, m+2}(\mu) \geq-r$.

Note that Corollary 4.11 is Theorem 4.4, the second symmetry.

To prove Theorem 4.9 and its corollaries, we present a family of maps,

$$
\mathfrak{D}_{r}^{k, m}: \mathcal{A} \rightarrow \mathcal{B}
$$

between the following two sets:

$$
\begin{aligned}
\mathcal{A}= & \{\text { partitions with } k \text { successive } m \text {-Durfee rectangles of non-zero } \\
& \text { width with }(k, m) \text {-rank at most }-r\} \\
\mathcal{B}= & \{\text { partitions with }(k, m+2) \text {-rank at least }-r\} .
\end{aligned}
$$

Procedure 4.12 (Generalized Dyson's map). Let $\lambda$ be a partition with $r_{k, m}(\lambda) \leq-r$.

Let $\alpha$ be the partition below the $k$ th successive $m$-Durfee rectangle and $\lambda^{1}, \lambda^{2}, \ldots, \lambda^{k}$ be the partitions to the right of the rectangles.

Let $N_{1}, N_{2}, \ldots, N_{k}$ be the widths of the $k$ successive $m$-Durfee rectangles and let

$$
p_{2}=N_{1}-N_{2}, p_{3}=N_{2}-N_{3}, \ldots, p_{k}=N_{k-1}-N_{k} .
$$

Say $\ell(\alpha)=t$. Then we obtain $k$ new partitions $\mu^{1}, \mu^{2}, \ldots, \mu^{k}$ by applying the insertion lemma so that

$$
\phi\left(t-r ; \lambda^{1}, \ldots, \lambda^{k}\right)=\left(\mu^{1}, \ldots, \mu^{k}\right) .
$$


Remove the first column from $\alpha$ (or equivalently subtract 1 from each part) to get a partition $\beta$.

Let $\mu$ be a new partition defined by having

- $k$ successive $(m+2)$-Durfee rectangles of widths $N_{1}-1, N_{2}-1, \ldots, N_{k}-1$,

- $\mu^{1}, \mu^{2} \ldots, \mu^{k}$ to the right of these rectangles, and

- $\beta$ below the $k$ th rectangle.

Then $\mathfrak{D}_{r}^{k, m}(\lambda)=\mu$.

When $k$ and $m$ are clear from context we will write $\mathfrak{D}_{r}$.

The essence of Dyson's map is to the remove the first column of a partition and, after adding or removing some boxes, make it the first row of the partition. Our map $\mathfrak{D}_{r}$, removes the first column of the partition below the successive Durfee rectangle and inserts it (minus $r$ boxes) into the sequence of partitions to the right of the Durfee rectangles. To do this, the shape of the Durfee rectangle is modified to be one row taller and one column narrower.

We will give three examples of applications of $\mathfrak{D}_{r}$ before giving the proof that $\mathfrak{D}_{r}$ is well-defined and gives a bijection between $\mathcal{A}$ and $\mathcal{B}$ that has the desired properties. See Figures [14, 15] and 16]

$\lambda$

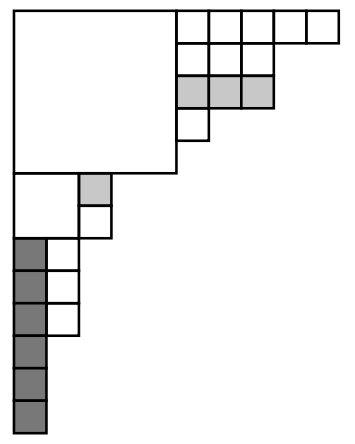

$\mathfrak{D}_{0}(\lambda)$

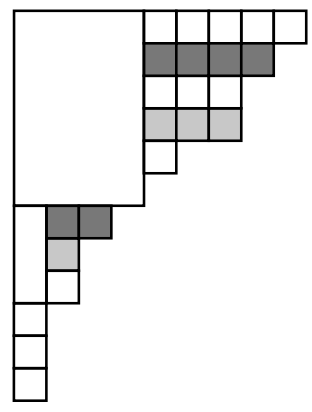

Figure 14. Applying $\mathfrak{D}_{0}^{2,0}$ to $\lambda=(10,8,8,6,5,3,3,2,2,2,1,1,1)$.

$\lambda$

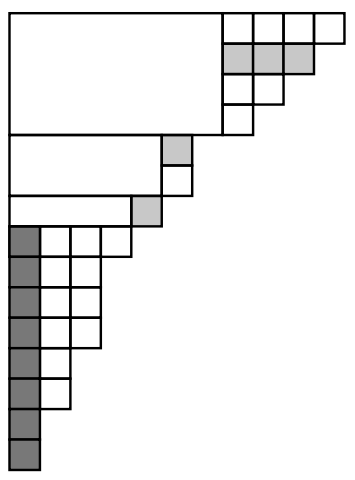

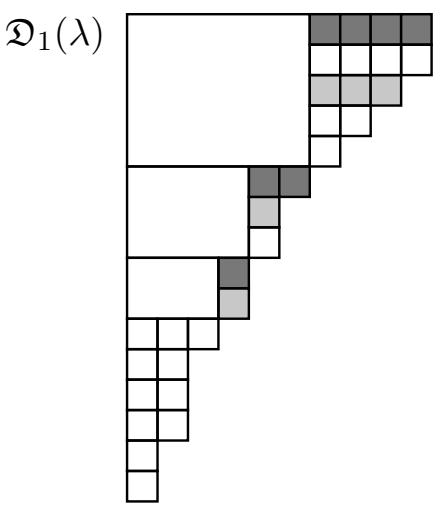

$\mathfrak{D}_{1}(\lambda)$

FiguRe 15. Applying $\mathfrak{D}_{1}^{3,-3}$ to $\lambda=(11,10,9,8,6,6,5,4,3,3,3,2,2,1,1)$. 

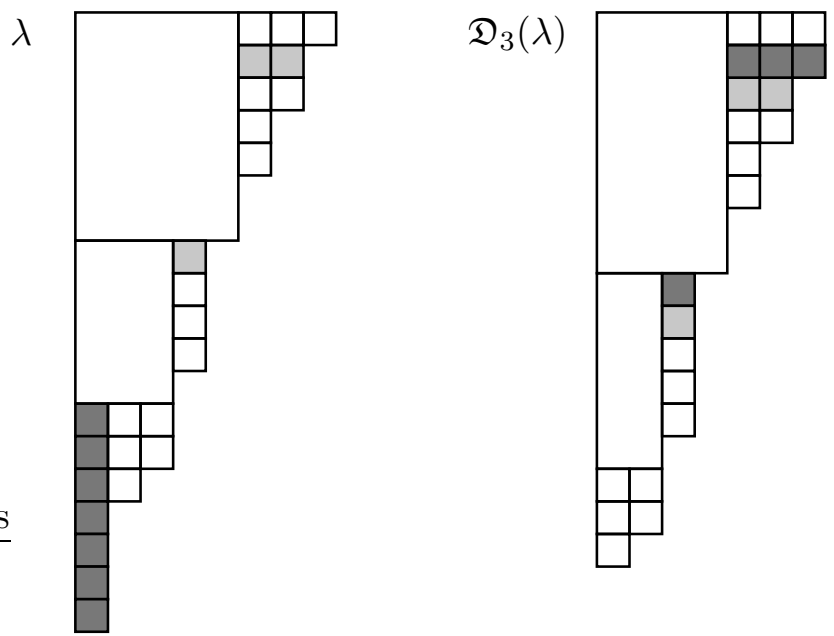

Figure 16. Applying $\mathfrak{D}_{3}^{2,2}$ to $\lambda=(8,7,7,6,6,5,5,4,4,4,4,3,3,3,2,1,1,1,1)$.

Proof of Theorem 4.9. To prove theorem 4.9, we show that $\mathfrak{D}_{r}^{k, m}$ is a bijection that changes rank and the other statistics of the partitions appropriately.

Consider a partition $\lambda$ with $k$ successive $m$-Durfee rectangles of widths $N_{1}, N_{2}, \ldots, N_{k}$ such that $r_{k, m}(\lambda) \leq-r$. Say

$$
\begin{gathered}
a_{k, m}(\lambda)=s, \\
b_{k, m}(\lambda)=t .
\end{gathered}
$$

First, we note that $\mu$ may have $(m+2)$-Durfee rectangles of width $N_{1}-1, N_{2}-1, \ldots, N_{k}-1$ since none of these integers are negative. (If $m=-2$, none of these can be zero either since the -2 -Durfee rectangles of $\lambda$ must have nonzero height.

Next we want to apply our insertion procedure and so we must verify that the conditions of Proposition 2.4 are satisfied. For each $2 \leq i \leq k, \lambda^{i}$ fits to the right of the $i$ th $m$-Durfee rectangle and below the $(i-1)$ st $m$-Durfee rectangle. As a consequence, its largest part satisfies $f\left(\lambda^{i}\right) \leq$ $N_{i}-N_{i-1}=p_{i}$ for $i \geq 2$ and $\ell\left(\lambda^{i}\right) \leq N_{i}+m$ for all $i \geq 1$.

We want to insert $t-r$ into $\lambda^{1}, \lambda^{2}, \ldots, \lambda^{k}$. Since $r_{k, m}(\lambda)=s-t \leq-r$, we get $A\left(\lambda^{i}, \lambda^{2}, \ldots, \lambda^{k} ; p_{2}, p_{3}, \ldots, p_{k}\right)=$ $a_{k, m}(\lambda)=s \leq t-r$ which is the required condition.

Now applying the lemma gives partitions $\mu^{1}, \mu^{2}, \ldots, \mu^{k}$ by inserting one part into each of $\lambda^{1}, \lambda^{2}, \ldots, \lambda^{k}$. Since we doing so gives $f\left(\mu^{i}\right) \leq p_{i}=\left(N_{i}-1\right)-\left(N_{i-1}-1\right)$ and $\ell\left(\mu^{i}\right) \leq N_{i}+m+1=\left(N_{i}-1\right)+(m+2)$. Hence we see that $\mu^{1}, \mu^{2}, \ldots, \mu^{k}$ will fit to the right of the first $k$ successive $(m+2)$-Durfee squares of width $N_{1}-1, N_{2}-1, \ldots, N_{k}-1$.

Finally, the largest part of $\beta$ is one less that the largest part of $\alpha$ and so $\beta$ fits under the $k$ th successive $(m+2)$-Durfee rectangle of $\mu$. (If $\alpha$ was empty, $\beta$ will be empty as well and will still fit.)

We may conclude that $\mu$ is a well-defined partition.

We must show that $\mathfrak{D}_{r}$ is a bijection. Notice that $\mathfrak{D}_{r}$ is reversible since

$$
\begin{aligned}
& \psi_{1}\left(\mu^{1}, \mu^{2}, \ldots, \mu^{k}\right)=t-r, \text { and } \\
& \psi_{2}\left(\mu^{1}, \mu^{2}, \ldots, \mu^{k}\right)=\left(\lambda^{1}, \lambda^{2}, \ldots, \lambda^{k}\right) .
\end{aligned}
$$


Hence we know $\lambda^{1}, \lambda^{2}, \ldots, \lambda^{k}$ and from this we can also recover $\alpha$ since we know $\beta$ and since $\ell(\alpha)=t=\psi_{1}+r$.

To show that $\mathfrak{D}_{r}$ is surjective for any partition $\mu$ with $k$ successive $(m+2)$-Durfee rectangles of widths $N_{1}-1, N_{2}-1, \ldots, N_{k}-1$ with $r_{k, m+2}(\mu) \geq-r$ we will construct a partition $\lambda$ such that $\mathfrak{D}_{r}(\lambda)=\mu$. Since $r_{k, m+2}(\mu) \geq-r$, there is some $t \geq 0$ such that, $\mu$ has $a_{k, m+2}(\mu)=t-r$ and $b_{k, m+2}(\mu) \leq t$. Let $\mu^{1}, \mu^{2}, \ldots, \mu^{k}$ be the partitions to the right of the $k$ successive $(m+2)$-Durfee rectangles and let $\beta$ be the partition below the $k$ th successive $(m+2)$-Durfee rectangle.

If we apply $\psi$ as above, we obtain partitions $\lambda^{1}, \lambda^{2}, \ldots, \lambda^{k}$ of the appropriate size to put to the right of $m$-Durfee rectangles of width $N_{1}, N_{2}, \ldots, N_{k}$. We can put a column to height $t=a_{k, m+2}(\mu)+r$ in front of $\beta$ since $b_{k, m+2}(\mu) \leq t$ This partition $\alpha$ fits below the $k$ th $m$-Durfee rectangle of width $N_{k}$. This gives a partition $\lambda$.

If we apply $\mathfrak{D}_{r}$ to $\lambda$ we are simply reversing the steps described above and so we get $\mathfrak{D}_{r}(\lambda)=\mu$. This shows that $\mathfrak{D}_{r}$ is surjective onto the set of partitions with $k$ successive $(m+2)$-Durfee rectangles.

We conclude that $\mathfrak{D}_{r}$ is indeed a bijection

It remains to check the required properties of $\mu$. We note that:

- by definition $\mu$ is a partition with $k$ successive $(m+2)$-Durfee rectangles of widths $N_{1}-1, N_{2}-1, \ldots, N_{k}-1$,

- since we inserted $t-r$ into $\lambda^{1}, \lambda^{2}, \ldots, \lambda^{k}$ we get

$$
a_{k, m}(\mu)=A\left(\mu^{1}, \mu^{2}, \ldots, \mu^{k} ; p_{2}, p_{3}, \ldots, p_{k}\right)=t-r,
$$

- since $\ell(\alpha)=t$ we get

$$
b_{k, m}(\mu)=\ell(\beta) \leq \ell(\alpha)=t, \text { and }
$$

- if $\lambda$ is a partition of $n, \mu$ is a partition of $n-r-k(m+1)$ since we remove a column of height $t$ from $\alpha$ to get $\beta$, insert $t-r$ into $\lambda^{1}, \lambda^{2}, \ldots, \lambda^{k}$ to get $\mu^{1}, \mu^{2}, \ldots, \mu^{k}$, and reduce the number of boxes in each of the $k$ successive Durfee rectangle by $m+1$.

For the two corollaries we note that $r_{k, m+2}(\mu)=a_{k, m+2}(\mu)-b_{k, m+2}(\mu) \geq t-r-t=-r$.

\section{Algebraic Derivation of the Generalized Schur identity}

We can now complete the proof of the generalized Schur identities. We proceed in a fashion similar to the algebraic steps of Dyson's proof of Euler's pentagonal number theorem using the two observations and the two symmetries of the previous section.

For every $j \in \mathbb{Z}$ let

$$
\begin{aligned}
& a_{j}=h\left(n-j r-\frac{j(j-1)}{2}-k\left(j m+j^{2}\right), k, m+2 j, \leq-r-j\right), \\
& b_{j}=h\left(n-j r-\frac{j(j-1)}{2}-k\left(j m+j^{2}\right), k, m+2 j, \geq-r-j+1\right) .
\end{aligned}
$$

In this notation, for $m+2 j>0$, the second observation, 4.2, gives us

$$
a_{j}+b_{j}=p\left(n-j r-\frac{j(j-1)}{2}-k\left(j m+j^{2}\right)\right) .
$$


For either $r+j>0$ or for $m+2 j \leq 0$, the second symmetry, 4.4 gives us

$$
a_{j}=b_{j+1} \text {. }
$$

Applying these multiple times we get

$$
\begin{aligned}
h(n, k, m, \leq-r)=a_{0}=b_{1} \\
\quad=b_{1}+\left(a_{1}-b_{2}\right)-\left(a_{2}-b_{3}\right)+\left(a_{3}-b_{4}\right)-\ldots \\
\quad=\left(b_{1}+a_{1}\right)-\left(b_{2}+a_{2}\right)+\left(b_{3}+a_{3}\right)-\left(b_{4}+a_{4}\right)+\ldots \\
\quad=\sum_{j=1}^{\infty}(-1)^{j-1} p\left(n-j r-\frac{j(j-1)}{2}-k\left(j m+j^{2}\right)\right) .
\end{aligned}
$$

This last identity holds if either $m \geq 0$ and $r \geq 1$ or if $m=r=0$.

In terms of the generating functions

$$
\begin{aligned}
& H_{k, m, \leq r}(q):=\sum_{n=0}^{\infty} h(n, k, m, \leq r) q^{n}, \text { and } \\
& H_{k, m, \geq r}(q):=\sum_{n=0}^{\infty} h(n, k, m, \geq r) q^{n},
\end{aligned}
$$

this gives, if either $m \geq 0$ and $r \geq 1$ or if $m=r=0$,

$$
H_{k, m, \leq-r}(q)=\frac{1}{(q)_{\infty}} \sum_{j=1}^{\infty}(-1)^{j-1} q^{j r+\frac{j(j-1)}{2}+k\left(j m+j^{2}\right)} .
$$

In particular, we have:

$$
\begin{aligned}
H_{k, 0, \leq 0}(q) & =\frac{1}{(q)_{\infty}} \sum_{j=1}^{\infty}(-1)^{j-1} q^{\frac{j(j-1)}{2}+k j^{2}}, \text { and } \\
H_{k, 0, \leq-1}(q) & =\frac{1}{(q)_{\infty}} \sum_{j=1}^{\infty}(-1)^{j-1} q^{\frac{j(j+1)}{2}+k j^{2}} .
\end{aligned}
$$

From the first symmetry 4.3 and the first observation 4.1 we note that

$$
H_{k, 0, \leq 0}(q)+H_{k, 0, \leq-1}(q)=H_{k, 0, \leq 0}(q)+H_{k, 0, \geq 1}(q)
$$

is the generating function for partitions with at least $k$ successive Durfee squares. We conclude:

$$
\begin{aligned}
\frac{1}{(q)_{\infty}} & \sum_{j=1}^{\infty}(-1)^{j-1} q^{\frac{j(j-1)}{2}+k j^{2}}+\frac{1}{(q)_{\infty}} \sum_{j=1}^{\infty}(-1)^{j-1} q^{\frac{j(j+1)}{2}+k j^{2}} \\
& =\frac{1}{(q)_{\infty}}-\sum_{n_{1}=0}^{\infty} \cdots \sum_{n_{k-1}=0}^{\infty} \frac{q^{N_{1}^{2}+N_{2}^{2}+\cdots+N_{k-1}^{2}}}{(q)_{n_{1}}(q)_{n_{2}} \cdots(q)_{n_{k-1}}}
\end{aligned}
$$

which implies the generalized Schur identities (2) and completes our proof of the generalized RogersRamanujan identities (1). 


\section{Connections to other Work}

6.1. Dyson's rank and proof of Euler's pentagonal number theorem. As mentioned in the introduction, this proof of the Rogers-Ramanujan identities follows the general form of Dyson's proof of Euler's pentagonal number theorem. More specifically, our proof of the generalized Schur identities (2) is a Dyson-style proof with a modified rank.

We generalized Dyson's rank by defining $(k, m)$-rank; his rank is our $(1,0)$-rank. The algebraic steps used to deduce the generalized Schur identities are the same as those used to deduce Euler's pentagonal number theorem. Moreover, our symmetries and corresponding bijections, $\mathfrak{C}^{k}$ and $\mathfrak{D}_{r}^{k, m}$ generalize conjugation and Dyson's map. More precisely, in the case $k=1$, we have:

$$
\begin{aligned}
r_{1, m}(\lambda) & =r(\lambda)-m, \\
\mathfrak{C}^{1} & =\text { usual conjugation, and } \\
\mathfrak{D}_{r}^{1, m} & =d_{-r-m} .
\end{aligned}
$$

This is not the first generalization of Dyson's rank that has been used to prove the RogersRamanujan identities. The notion of successive rank can also be used to give a combinatorial proof of the Rogers-Ramanujan identities and their generalizations by a sieve argument (see [1, 5, 11]). However, this proof does not use the notion of successive Durfee squares but rather involves a different combinatorial description of the partitions on the left hand side of the Rogers-Ramanujan identities. This other generalization of Dyson's rank was kindly brought to our attention by George Andrews.

6.2. Bressoud and Zeilberger. A list of work connected to this proof is not complete without mentioning the bijective Rogers-Ramanujan proof of Bressoud and Zeilberger. In [13, 14, they give a bijection proving Andrews' generalization of the Rogers-Ramanujan identities (II) based on the involution principle and Bressoud's short Rogers-Ramanujan proof [12. One of their maps, $\Phi$ in [14, acts similarly to our maps $\mathfrak{D}_{r}^{k, m}$ for certain $k, m$, and $r$. Unfortunately, due to the complexity of their proofs we do not give a formal connection. The fact that these maps are somewhat similar does however have consequences for the question in the last section.

6.3. Garvan and Berkovich. Garvan has also defined a generalized notion of rank for partitions with multiple Durfee squares [19. Though different from our definition, his rank leads to the same generating function for partitions with rank at most $-r$ as we derived the previous section. Based on this generating function, in [6], Berkovich and Garvan ask for a symmetry similar to Dyson's "new symmetry" for Garvan's generalized rank and for a Dyson-style proof of their generating function. They note that it "turned out to be very difficult to prove in a combinatorial fashion."

We will explain the relationship between our generalization of rank and Garvan's definition, and the two symmetries associated with both definitions. We will also be able to show why the Dyson-style proof sought by Berkovich and Garvan turns out to be difficult to find.

6.3.1. Garvan's rank and conjugation. Recall that for a partition $\lambda$ with $k$ successive Durfee squares, we denote the partitions to the right of these Durfee squares by $\lambda^{1}, \lambda^{2}, \ldots, \lambda^{k}$ and the partition below the $k$ th Durfee square by $\alpha$. 
Definition 6.1 (Garvan, [19]). Let $\lambda$ be a partition with at least $k$ successive Durfee squares, where the $k$ th Durfee square has size $N_{k}$. Define

$$
\begin{aligned}
& g a_{k}(\lambda)=\text { the number of columns of } \lambda^{1} \text { whose length } \leq N_{k}, \text { and } \\
& g b_{k}(\lambda)=\ell(\alpha) .
\end{aligned}
$$

Also, define

$$
g r_{k}(\lambda)=g a_{k}(\lambda)-g b_{k}(\lambda) .
$$

We will call $g r_{k}(\lambda)$ Garvan's rank.

Garvan called $\operatorname{gr}_{k}(\lambda)$ the $(k+1)$-rank of $\lambda$. See Figure 17 for an example.

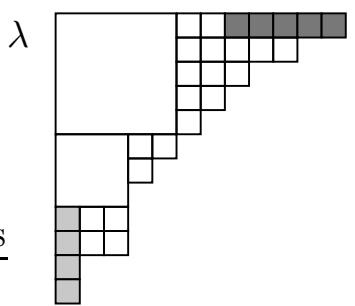

Figure 17. Partition $\lambda=(12,10,8,7,6,5,4,3,3,3,1,1)$ has $g a_{2}(\lambda)=5, g b_{2}(\lambda)=4$, and $g r_{2}(\lambda)=5-4=1$.

Garvan also described a very natural conjugation for partitions with $k$ successive Durfee squares. For any partition $\lambda$ with $k$ successive Durfee squares of size $N_{1}, N_{2}, \ldots, N_{k}$, let

- $\alpha$ be the partition below the $k$ th Durfee square, and

- $\beta^{\prime}$ be the partition consisting of columns sitting to the right of the first Durfee square of $\lambda$ whose length is $\leq N_{k}$.

The conjugate is obtained by replacing $\alpha$ and $\beta^{\prime}$ by $\beta$ and $\alpha^{\prime}$, respectively. Note that conjugation is clearly an involution that sends Garvan's rank of a partition to its negative. See Figure 18.
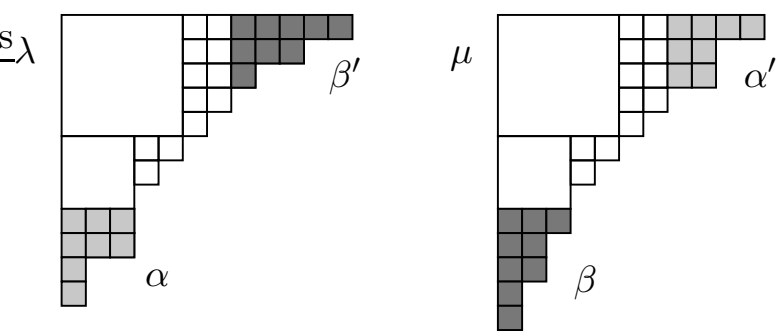

Figure 18. Partition $\lambda=(12,10,8,7,6,5,4,3,3,3,1,1)$ and its Garvan conjugate $\mu=(11,9,9,7,6,5,4,3,3,2,2,1,1)$.

6.3.2. Relationship between $(k, m)$-rank and Garvan's ranks. One first theorem tells us that $(k, 0)$ rank and Garvan's ranks have the same distribution on partitions of $n$.

Theorem 6.2. For any $k, n, s, t \in \mathbb{Z}$, the number of partitions $\lambda$ of $n$ with $k$ successive Durfee squares, of size $N_{1}, N_{2}, \ldots, N_{k}$, with $a_{k, 0}(\lambda)=s$ and $b_{k, 0}(\lambda)=t$ is equal to the number of partitions $\mu$ of $n$ with $k$ successive Durfee squares, of size $N_{1}, N_{2}, \ldots, N_{k}$, with $g a_{k}(\mu)=s$ and $g b_{k}(\mu)=t$. 
In particular, for any $k, n, r \in \mathbb{Z}$, the number of partitions $\lambda$ of $n$ with $r_{k, 0}(\lambda)=r$ is equal to the number of partitions $\mu$ of $n$ with $g r_{k}(\mu)=r$.

We will give an outline of the proof of this theorem to show the relationship between the two ranks; the full proof of this theorem is found in [7].

For both definitions, consider the squares of the Young diagram that are exchanged by the corresponding conjugation and the squares that are not exchanged by the corresponding conjugation. See Figure 19 .
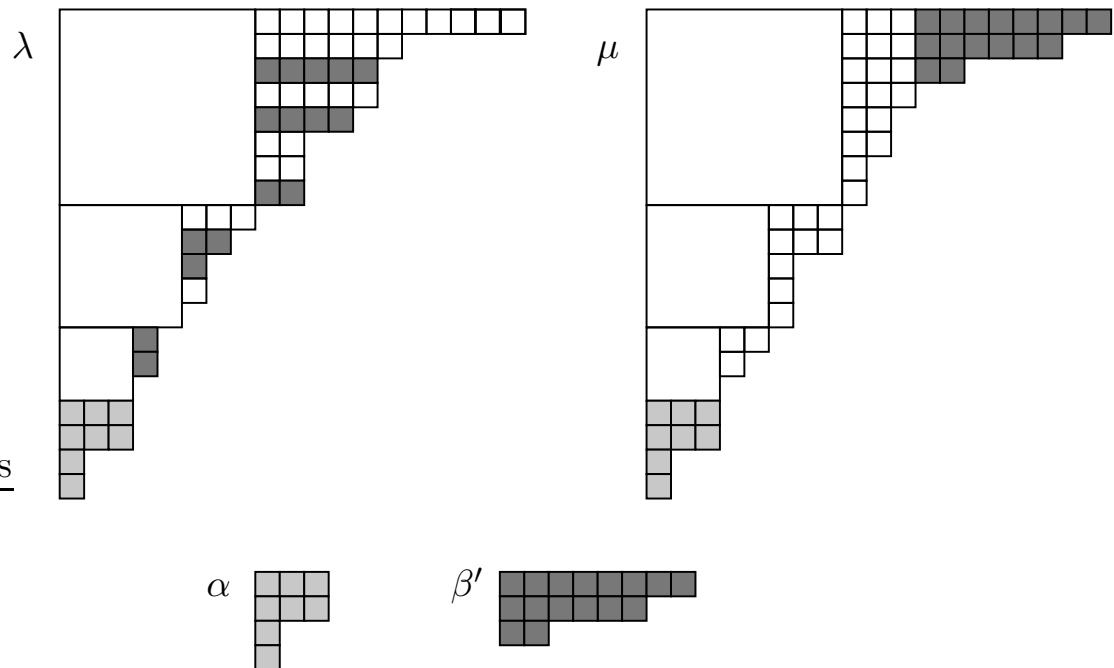

FiguRE 19. The shaded squares are those exchanged by conjugation.

The squares of $\mu$ that are exchanged by Garvan's conjugation make up $\alpha$, the partition below $k$ th Durfee square, and $\beta^{\prime}$, the partition made up of columns having height less than or equal to $N_{k}$ in $\lambda^{1}$, the partition to the right of the first Durfee square. In our conguation, $\mathfrak{C}^{k}$, the squares of $\lambda$ that are exchanged are (in the notation from section 4.1) $\alpha$, the partition below $k$ th Durfee square, and the partition $\beta^{\prime}$ obtained by iteratively removing selected parts from $\lambda^{1}, \lambda^{2}, \ldots, \lambda^{k}$. Recall that this $\beta^{\prime}$ has at most $N_{k}$ parts just as the $\beta^{\prime}$ from Garvan's conjugation.

Now consider the squares that are not exchanged by the corresponding conjugation. We are left with two different sets of partitions. In the case of Garvan's rank, the partitions have no part below the $k$ th Durfee square and no column whose length is less than or equal to $N_{k}$ to the right of the first Durfee square. In the case of our rank, the partitions have no part below the $k$ th Durfee square and no part to the right of the bottom $N_{k}$ rows of each Durfee square. See Figure 20 for an example of these types of partitions.

From this point, the proof may proceed in two ways: bijectively or by considering the generating function keeping track of $g a_{k}, g b_{k}, g r_{k}$ and size of the partitions and $a_{k, 0}, b_{k, 0}, r_{k, 0}$ and size of the partitions depending on the case.

In both cases, the squares that are exchanged by conjugation match up, $\alpha$ with $\alpha$ and $\beta^{\prime}$ with $\beta^{\prime}$. The squares that are not exchanged by conjugation also correspond. Notice that these latter squares do not contribute to any of the statistics other than size of the partitions. Therefore we only need to show that there is the same number of either type of a given size $n$ with Durfee squares of size $N_{1}, \ldots, N_{k}$. By generating functions this is a simple exercise involving $q$-binomials. Alternatively, a bijection can be found based a map due to Franklin, from section 20 of [24]. 

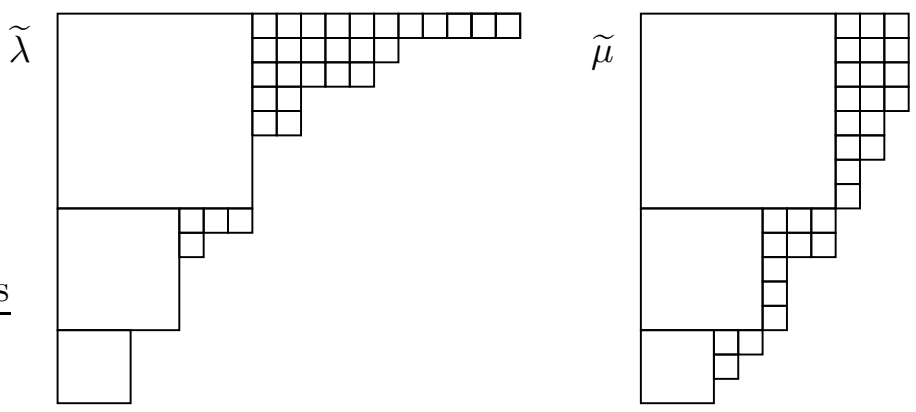

FiguRE 20. Shaded parts have been removed to leave only the parts untouched by conjugation.

This setup also shows us that Garvan's conjugation and our conjugation are related in a natural way since we match up exactly the squares that are exchanged by either conjugation.

6.3.3. A version of Dyson's map for Garvan's rank. Since Garvan's rank and $(k, 0)$-rank are equidistributed on partitions of a given size and since we can relate the conjugations associated with each rank, it is natural look for a Dyson-like map similar to ask our second symmetry, $\mathfrak{D}_{r}^{k, m}$, for Garvan's rank.

Of course, it is possible to map $\mathfrak{D}_{r}^{k, m}$ through the bijection refered to in the previous section and get some (akwardly described) map that deals with Garvan's rank. However a few subtleties arise. Our bijection $\mathfrak{D}_{r}^{k, m}$ involves both the $(k, m)$-rank of a partition and the $(k, m+2)$-rank of a partition. Moreover, it changes the Durfee rectangle structure of the partition. To give a version of Dyson's map for Garvan's notion of rank based on our work neccessarily involves a more general notion of Garvan's rank for partitions with successive Durfee rectangles (as opposed to only squares) and such a Dyson-like bijection neccessarily changes the Durfee rectangle structure of the partition.

In fact, this last point is the reason that, as Berkovich and Garvan stated, it is difficult to find a Dyson's map for Garvan's rank. It turns out that even on fairly small examples numerical evidence shows that no Dyson-like bijection for Garvan's rank can maintain the Durfee square structure of the partitions.

\section{Further QUESTION}

Andrews generalized the Rogers-Ramanujan identities further than the identities (10) we have proved here. For $1 \leq a \leq k$, he proved that:

$$
\sum_{n_{1}=0}^{\infty} \cdots \sum_{n_{k-1}=0}^{\infty} \frac{q^{N_{1}^{2}+N_{2}^{2}+\cdots+N_{k-1}^{2}+N_{a}+\cdots+N_{k-1}}}{(q)_{n_{1}}(q)_{n_{2}} \cdots(q)_{n_{k-1}}}=\prod_{\substack{n=1 \\ n \neq 0, \pm a(\bmod 2 k+1)}}^{\infty} \frac{1}{1-q^{n}}
$$

and provided a combinatorial interpretation of the left hand side as a Durfee dissection using both Durfee squares and 1-Durfee rectangles [2, 4]. Further generalizations that lend themselves to similar interpretations have been given by Bressoud as well [9, 10 and by Garrett, Ismail, and Stanton [18]. Can our proof be extended to prove these identities?

It is fairly simple to extend our definition of $(k, m)$-rank and obtain bijections proving a first and second symmetry for these partitions in Andrews' identity. However, in this case, the second symmetry is not enough to determine the generating function for partitions with rank at most $-r$. In order to complete the proof, a new idea is required. 
On the other hand, there is evidence that our proof will not extend. The Rogers-Ramanujan bijection given by Bressoud and Zeilberger [13, 14 is a combinatorialization of a short proof of Bressoud [12] in which he proves the following generalization of Schur's identity:

$$
\begin{aligned}
\sum_{s_{1}=0}^{\infty} & \cdots \sum_{s_{k}=0}^{\infty} \frac{q^{s_{1}^{2}+s_{2}^{2}+\cdots+s_{k}^{2}}}{(q)_{N-s_{1}}(q)_{s_{1}-s_{2}} \cdots(q)_{s_{k-1}-s_{k}}(q)_{2 s_{k}}}(-x q ; q)_{s_{k}}\left(-x^{-1} ; q\right)_{s_{k}} \\
& =\frac{1}{(q)_{2 N}} \sum_{j=-\infty}^{\infty} x^{j} q^{\frac{(2 k+1) j^{2}+j}{2}}\left[\begin{array}{c}
2 N \\
N-m
\end{array}\right]_{q} .
\end{aligned}
$$

However, this generalization is quite different from Andrews' generalization given above. Since our map $\mathfrak{D}_{r}^{k, m}$ acts similarly to one of Bressoud and Zeilberger's maps, it may be that our proof is more likely to extend to this generalization rather than equation (3).

If our proof were extended to either case, this would also give a proof of the second RogersRamanujan identity.

\section{ACKNOWLEDGMENTS}

The author is grateful to George Andrews, Igor Pak, and Richard Stanley for their support, encouragement, and helpful comments.

\section{REFERENCES}

[1] George E. Andrews. Sieves in the theory of partitions. Amer. J. Math., 94:1214-1230, 1972.

[2] George E. Andrews. An analytic generalization of the Rogers-Ramanujan identities for odd moduli. Proc. Nat. Acad. Sci. USA, 71:4082-4085, 1974.

[3] George E. Andrews. The Theory of Partitions. Addison-Wesley, Reading, MA, 1976.

[4] George E. Andrews. Partitions and Durfee dissection. Amer. J. Math., 101:735-742, 1979.

[5] George E. Andrews, R. J. Baxter, D. M. Bressoud, W. H. Burge, P. J. Forrester, and G. Viennot. Partitions with prescribed hook differences. European J. Combin., 8:341-350, 1987.

[6] Alexander Berkovich and Frank G. Garvan. Some observations on Dyson's new symmetries of partitions. J. Combin. Theory Ser. A, 100:61-93, 2002.

[7] Cilanne E. Boulet. Partition identity bijections related to sign-balance and rank. PhD thesis, Massachusetts Institute of Technology, Department of Mathematics, April 2005.

[8] Cilanne E. Boulet and Igor Pak. A new combinatorial proof of the Rogers-Ramanujan and Schur identities. J. Combin. Theory Ser. A, 113(6):1019-1030, 2006.

[9] David M. Bressoud. A generalization of the Rogers-Ramanujan identities for all moduli. J. Combin. Theory Ser. A, 27:64-68, 1979.

[10] David M. Bressoud. An analytic generalization of the Rogers-Ramanujan identities with interpretation. $Q$. J. Math., 31:385-399, 1980.

[11] David M. Bressoud. Extensions of the partition sieve. J. Number Theory, 12:87-100, 1980.

[12] David M. Bressoud. An easy proof of the Rogers-Ramanujan identities. J. Number Theory, 16:235-241, 1983.

[13] David M. Bressoud and Doron Zeilberger. A short Rogers-Ramanujan bijection. Discrete Math., 38:313-315, 1982.

[14] David M. Bressoud and Doron Zeilberger. Generalized Rogers-Ramanujan bijections. Adv. Math., 78:42-75, 1989.

[15] Freeman J. Dyson. Some guesses in the theory of partitions. Eureka (Cambridge), 8:10-15, 1944.

[16] Freeman J. Dyson. A new symmetry of partitions. J. Combin. Theory, 7:65-61, 1969.

[17] Freeman J. Dyson. A walk through Ramanujan's garden, pages 7-28. Ramanujan revisited. Academic Press, Boston, MA, 1988.

[18] K. Garrett, M. E. Ismail, and Dennis Stanton. Variants of the Rogers-Ramanujan identities. Adv. in Appl. Math., 23:274-299, 1999.

[19] Frank G. Garvan. Generalizations of Dyson's rank and non-Rogers-Ramanujan partitions. Manuscripta Math., 84:343-359, 1994.

[20] Igor Pak. On Fine's partition theorems, Dyson, Andrews, and missed opportunities. Math. Intelligencer, 25(1):1016, 2003. 
[21] Igor Pak. Partition bijections, a survey. Ramanujan J., 12(1), 2006.

[22] L. J. Rogers. Second memoir on the expansion of certain infinite products. Proc. London Math. Soc., 25:318-343, 1894.

[23] I. Schur. Ein Beitrag zur Additiven Zhalentheorie und zur Theorie der Kettenbrüche. S.-B. Preuss. Akad. Wiss. Phys. Math. Klasse, pages 302-321, 1917.

[24] J. J. Sylvester and F. Franklin. A constructive theory of partitions, arranged in three acts, an interact and an exodion. Amer. J. Math., 5:251-330, 1882.

[25] E. M. Wright. An enumerative proof of an identity of Jacobi. J. London Math. Soc., 40:55-57, 1965. 